\title{
Description of a Class of Superstring Compactifications Related to Semi-Simple Lie Algebras
}

\author{
D. G. Markushevich, M. A. Olshanetsky, and A. M. Perelomov \\ Institute of Theoretical and Experimental Physics, Bol. Cheremushkinskaya, dom 25, \\ SU-117259 Moscow, USSR
}

\begin{abstract}
A class of vacuum configurations in the superstring theory obtained by compactification of physical dimensions from ten to four is constructed. The compactification scheme involves taking quotients of tori of semisimple Lie algebras by finite symmetry group actions. The complete list of such configurations arising from actions by a Coxeter transformation is given. Some topological invariants having physical interpretations are calculated.
\end{abstract}

\section{Introduction}

One of the most important aspects of the Green-Schwarz superstring [1] and the heterotic string [2] theories is the compactification of the six space-time dimensions. One possible solution of consistency for the theory is that the internal six-dimensional space be Kählerian and Ricci-flat, i.e. the so-called Calabi-Yau space (CYS). The structure of CYS is essential in determining the form of the lowenergy lagrangian of the theory. So breaking the gauge group by the holonomy group of the CYS leads to a model in which the number of generations is equal to $1 / 2|\chi|, \chi$ being the Euler characteristic of the CYS [3] (the more deep breaking pattern links the number of generations to the third Chern class of some vector bundle on the CYS [4]). The number of massless scalar modes is expressed in terms of Betti numbers of the CYS [3]. The resulting breaking of the great unification gauge group depends also on the fundamental group $\pi_{1}$ (CYS) [5]. The Yukawa couplings are essentially topological as well; they are simply intersection indices of four-dimensional surfaces in CYS [6]. At present some examples of CYS are known, but there is no general classification. The further progress in superstring theory depends on our understanding the structure of CYS. It is useful to have the largest possible list of CYS containing simple examples as well as phenomenologically acceptable cases. A more detailed study of superstring dynamics will indicate the one which leads to the observed spectrum of chiral fermions.

In the present paper we construct new examples of CYS using one of the known methods [7]. It consists of the following. Let us take a three-dimensional complex torus $T_{\mathbb{C}}^{3}$. This is a simplest CYS since its group of holonomy is trivial. Therefore 
the compactification on the torus will not lead to the gauge symmetry breaking. However in some cases it becomes possible to "spoil" it in such a way that the "spoiled" torus has all of $\mathrm{SU}_{3}$ as its holonomy group. For this it is necessary that the torus have some additional symmetries. Suppose that there is a finite subgroup $\Gamma$ of $\mathrm{SU}_{3}$ acting linearly on the torus. The fixed points of this action give rise to singularities in the quotient space $K_{0}=T_{\mathbb{C}}^{3} / \Gamma$, which is called an orbifold. The algebraic-geometrical tools make it possible to smooth out these singularities (or to resolve them) [8]. After desingularization we get a smooth manifold $K$. The choice of the subgroup $\Gamma$ and of the procedure of desingularization depends on the assumption that the resulting manifold be a CYS, i.e. a Kählerian and Ricci-flat manifold. The class of CYS constructed in this way is extremely convenient for studying the behaviour of the superstring. One can consider the string propagating in the singular orbifold $K_{0}$ whose structure is better understandable; one gets from such considerations the correct answers to some questions (for example, the one concerning the number of generations [9]).

The initial tori in this paper are real tori $T_{\mathbb{R}}^{6}$ on which large symmetry groups act. They all $^{1}$ are maximal tori of semisimple Lie groups of rank 6 . The Weil group, and even its extension generated by exterior automorphisms of the Dynkin diagram, acts on such tori (see [10]). As the real reflections, generating the Weil group, are not $\mathrm{SU}_{3}$-transformations, the Weil group is not contained in $\mathrm{SU}_{3}$. But it has some subgroups contained in $\mathrm{SU}_{3}$. In particular, the cyclic subgroup generated by a Coxeter transformation [11] proves to be such a subgroup. There is sufficiently much known about Coxeter transformations to describe explicitly the construction of CYS and evaluate some of their topological invariants. We give the list of all the semisimple Lie algebras of rank 6, such that their quotients under Coxeter automorphisms desingularize to give a CYS.

The paper consists of five parts (not counting the introduction). In the first one we give some generalities of CYS and expose the main construction. At the end we give the table of all the CYS which can be obtained by our method. This table contains 14 examples of CYS, of which only the first one has been known before [3]. For resolving singularities we use methods of toric geometry. We describe the toric desingularization method in the Appendix.

The next section contains calculations of some topological invariants of the constructed CYS. At first we give a proof of the formula for the Euler characteristic suggested in [9]. With the help of this formula we evaluate the Euler characteristics of CYS from our table. In all the cases excluding the one known before it turns out to be 48. It is interesting to note that all the values of the Euler characteristic are multiples of 24 (namely, 48 and 72). It is not clear whether it is an accidental or a natural phenomenon. If we apply the same method to the two-dimensional case we shall obtain the three types of the so-called Kummer K3-surfaces [8], all of which have the Euler characteristic 24. In this context, observe that our CYS have the same Euler characteristic as that of the fibre-bundle over the projective line $\mathbb{P}^{1}$ with a $K 3$-surface as a fiber [the Euler characteristic is multiplicative in fiberbundles: $\left.\chi(K)=\chi\left(\mathbb{P}^{1}\right) \cdot \chi(K 3)=2 \cdot 24=48\right]$. Indeed, most of our CYS have a structure of a "bundle" over $\mathbb{P}^{1}$ whose generic fiber is a $K 3$-surface, but this

${ }^{1}$ Note that not all complex tori with discrete symmetries from $\mathrm{SU}_{3}$ are obtained in this way 
"bundle" has degenerate singular fibers which may even contain several components (mutually intersecting complex surfaces) and so the multiplicativity of $\chi$ can't be applied.

We also compute the fundamental group. These computations can be done in the orbifold limit, because the desingularization does not change the fundamental group.

A separate section is devoted to the computation of Yukawa couplings. This computation, in contrast to the above, requires the knowledge of the explicit resolution of singularities, and we go through it for one of our CYS. Observe that most Yukawa couplings vanish. Analysis of other examples shows that in general $85-95 \%$ of them vanish.

Finally, in the closing section we consider possible generalizations of the suggested construction and discuss some other topological invariants having a phenomenological meaning.

\section{The Main Construction}

\section{Generalities on Calabi-Yau Spaces}

By a Calabi-Yau space (CYS) we mean a compact complex manifold $K$ admitting a Kählerian metric with zero Ricci tensor. By the Yau theorem [12], the existence of a Kähler Ricci-flat metric is equivalent to the vanishing of the real first Chern class: $c_{1}^{\mathbb{R}}(K)=0$. Note the first Chern class in $H_{D R}^{2}(K, \mathbb{R})$ is represented by the differential two-form

$$
\alpha=R_{i j} d x^{i} \wedge d x^{j},
$$

where $R_{i j}$ is the Ricci curvature tensor. The condition $c_{1}^{\mathbb{R}}(K)=0$ means that the 2form $\alpha$ is exact, i.e. that $\alpha=d \beta$ for some $\beta$. Yau's theorem says that for an appropriate choice of a metric the form $\alpha$ is identically zero. Such a metric is uniquely determined by its cohomology class in the Hodge group $H^{1,1}(K, \mathbb{C})$.

From known results on CYS one can deduce that $c_{1}^{\mathbb{R}}(K)=0$ iff the canonical bundle $\Omega_{K}$ has finite order in the Picard group of $K$, i.e. $\Omega_{K}^{\otimes m} \simeq \mathcal{O}_{K}, \mathcal{O}_{K}$ being the trivial line bundle (see, for example, [13]). We recall that by the canonical bundle one means the vector bundle of holomorphic differential forms of the highest degree on $K$, or "holomorphic" volume elements. This criterion is the most convenient for our purposes, because holomorphic objects (such as meromorphic sections of $\Omega_{K}$ ) are easier to deal with than those built from a metric [such as $c_{1}^{\mathbb{R}}(K)$ ]. If $\Omega_{K}$ has a non-zero meromorphic section $\omega$, then we have in each open set $U$, with coordinates $\left(z_{1}, \ldots, z_{n}\right)$, the representation $\omega=g\left(z_{1}, \ldots, z_{n}\right) d z_{1} \wedge \ldots \wedge d z_{n}$, and the divisor of $\omega$ is defined by defining its restrictions to coordinate open sets: $(\omega)_{U}=(g)_{U}$. The triviality of $\Omega_{K}^{\otimes m}$ means that $m(\omega)=(f)$ for some meromorphic function $f(m \in \mathbb{Z}, m \geqq 1)$. In particular, $\Omega_{K}$ is trivial $(m=1)$ if there exists a form without poles or zeros, or such that $(\omega)=0$.

It is appropriate to remark here about the relationship with the holonomy group. If $K$ is an arbitrary compact riemannian manifold with a metric $g$, then we have the Riemann connection on the tangent bundle $T K$. Its holonomy group, referred to a point $x_{0} \in K$, is the group of automorphisms of the tangent space, 
$T_{x_{0}} K$, which send each tangent vector at $x_{0}$ to the result of its parallel translation along some closed path starting and ending at $x_{0}$. Generically $H \subset O(n)$, where $n=\operatorname{dim} K$. If $H \subset U(p), n=2 p$, then there is a complex structure on $K$, and $g$ is kählerian with respect to this complex structure. Let $H_{0}$ denote the component of the identity in $H$. If furthermore $H_{0} \subset \mathrm{SU}(p)$, then the Ricci tensor of the metric $g$ vanishes identically. The conditions $R_{i j}=0$ and $H \subset \mathrm{SU}(p)$ are equivalent. A kählerian manifold for which $H_{0}=\mathrm{SU}(p)$ is called an irreducible special unitary manifold. If at last $H_{0} \subset \mathrm{Sp}(r) \subset \mathrm{SU}(2 r)$ (here $n=2 p=4 r$ ), then $K$ is a symplectic manifold, and if the equality occurs, $H_{0}=\mathrm{Sp}(r)$, then $K$ is an irreducible symplectic manifold. The decomposition theorem for Ricci-flat manifolds [13] says that some non-ramified finite covering of $K$ is holomorphically isometric to the direct product $T^{k} \times V_{1} \times \ldots \times V_{q} \times X_{1} \times \ldots \times X_{\ell}$, where $T^{k}$ is a $k$-dimensional complex torus (whose holonomy group is trivial), $V_{i}$ are irreducible special unitary manifolds, and $X_{j}$ are irreducible symplectic ones, $V_{j}$ and $X_{j}$ being compact and simply connected. This decomposition corresponds to the decomposition of the representation of $H_{0}$ on $T_{x_{0}} K$ into the direct product of fundamental representations

$$
H_{0} \simeq 1_{K} \times \mathrm{SU}\left(m_{1}\right) \times \ldots \times \mathrm{SU}\left(m_{k}\right) \times \mathrm{Sp}\left(r_{1}\right) \times \ldots \times \mathrm{Sp}\left(r_{\ell}\right) .
$$

We will mention some corollaries. Firstly, for Ricci-flat manifolds we have $\left[H: H_{0}\right]<\infty$. Secondly, if $H$ has no invariants in $T_{x_{0}} K$, then $\pi_{1}(K)$ is a finite group. In particular the fundamental groups of irreducible special unitary or symplectic manifolds are finite. The fact that the fundamental group of $K$ is infinite implies that $K$ is a locally trivial fibration by tori which becomes trivial after pull-back to some finite non-ramified covering.

Thirdly, from the decomposition theorem we can deduce the above statement on triviality of $\Omega_{K}^{\otimes m}$ for some $m \geqq 1$. This follows from the Bochner principle:

Bochner Principle. Let $K$ be a compact kählerian manifold having zero Ricci curvature tensor. Then each holomorphic tensor field on $K$ is parallel with respect to the Kähler connection and, consequently, its value at $x_{0}$ is invariant under $H$. Conversely, every holomorphic tensor at $x_{0}$ which is invariant under $H$ has a unique extension to a parallel holomorphic tensor field on $K$.

Indeed, each tensor $\tau_{x_{0}}$ from $\operatorname{det} T_{x_{0}}^{*} K=\Lambda_{\mathbb{C}}^{n} T_{x_{0}}^{*} K$ is invariant under $H_{0}$, since elements of $H_{0}$ have determinant 1. As $\left[H: H_{0}\right]<\infty$, the tensor $\tau_{x_{0}}^{m}$ is invariant under $H$, where $m$ is the exponent of the finite group $H / H_{0}$ (i.e. $g^{m}=1$ for all $\left.g \in H / H_{0}\right)$. According to the Bochner principle $\tau_{x_{0}}^{m}$ extends to a holomorphic tensor field $\tau^{m}$; it yields the trivialization of $\Omega_{K}^{\otimes m}=\left(\operatorname{det} T_{x_{0}}^{*} K\right)^{\otimes m}$, since it has no zeros or poles on $K$ (if it had, it could not be parallel with respect to a metric connection).

One-dimensional CYS are complex tori $T_{\mathbb{C}}^{1}$ (algebraic geometers say "elliptic curves" instead). For tori $m=1$. Two-dimensional CYS are $T_{\mathbb{C}}^{2}$, or $K 3$-surfaces, or Enriques surfaces [8]. For surfaces, $m \leqq 2$ and $m=2$ only for Enriques surfaces. For three-dimensional CYS $m \leqq 66$, and there are examples with $m=66$ [13]. To within finite non-ramified coverings, three-dimensional CYS are divided into three classes: (1) three-dimensional complex tori (having the trivial holonomy group); (2) the products $E \times S$ of an elliptic curve $E$ and a $K 3$-surface $S$ [here the holonomy group is $\mathrm{SU}(2)=\mathrm{Sp}(1)]$; (3) simply connected irreducible special unitary manifolds 
[with the holonomy group SU(3)]. The structure of the classes (1), (2) is wellknown. As for manifolds from the third class, there are only some examples known. Below we describe a construction giving a series of new examples of CYS from the third class.

\section{Description of the Construction}

The initial manifold is the real six-dimensional torus $T^{6}=\mathbb{R}^{6} / L$, where $L$ is a complete period lattice of the torus in $\mathbb{R}^{6}$. We consider tori whose period lattices are root lattices of semisimple Lie algebras of rank 6 . Such a lattice is defined by basic roots $\alpha_{1}, \ldots, \alpha_{6}$. The Weil group $W$, generated by the reflections $s_{j}, j=1, \ldots, 6$ in the hyperplanes orthogonal to the simple roots $\alpha_{j}$, acts on the lattice. This action defines the action of $W$ on the torus $T^{6}$. Consider the group $\Gamma \subset W$ generated by the so-called Coxeter element $c=s_{1} \cdot s_{2} \cdot \ldots \cdot s_{6}$ :

$$
\Gamma=\left\{1, c, \ldots, c^{h-1}\right\} \quad\left(c^{h}=1\right),
$$

where $h$ is the Coxeter number (the order of $c$ ). Instead of $s_{1} \ldots s_{6}$, one can also consider the generalized Coxeter element twisted by an exterior automorphism of the Dynkin diagram [11].

We obtain CYS by taking quotients of a three-dimensional complex torus under $\Gamma$. In order to demonstrate the idea of taking quotients of a torus, we describe a model example, the one considered in [3]. (Let $n=3$. In particular, $n=1 \ldots$.)

Consider the lattice $L$ of roots of the algebra $A_{2}$ in the complex plane $\mathbb{C}$. It is generated by two basic roots, $\omega=\exp 2 \pi i / 3$ and 1 . The action of $c$ has the form $z \rightarrow \omega z$. A point $z_{0} \in \mathbb{C}$ determines the point of the torus $T=\mathbb{C} / L$, which will be denoted by $\left[z_{0}\right]$. The point $\left[z_{0}\right]$ is fixed by $c$ if there exists a point $\gamma \in L, \gamma=a+b \omega$ $(a, b \in \mathbb{Z})$ such that

$$
c z_{0}=z_{0}+\gamma .
$$

It is obvious that in order to determine all the fixed points, we can restrict ourselves with examining points of any basic parallelogram $\Pi$; let us take, for example, the one spanned by 1 and $\omega$ (see Fig. 1).

One can easily verify that there are three fixed points of $c$ on the torus $T$, whose representatives in $\Pi$ are:

$$
\alpha_{1}=0, \quad \alpha_{2}=\frac{1}{3}(2+\omega), \quad \alpha_{3}=\frac{1}{3}(1+2 \omega) .
$$

We have

$$
c \alpha_{2}=\alpha_{2}-1, \quad c \alpha_{3}=\alpha_{3}+(1-\omega) .
$$

The hatching covers the fundamental domain of the symmetry group generated by $L$ and $c$. To obtain $T /\langle c\rangle$, one should fold the rhombus $\left[\alpha_{1}, \alpha_{2}, 1+\omega, \alpha_{3}\right]$ along the diagonal $\alpha_{2} \alpha_{3}$ and glue together the sides $\alpha_{1} \alpha_{2}$ and $\alpha_{2}(1+\omega)$, as well as $\alpha_{1} \alpha_{3}$ and $\alpha_{3}(1+\omega)$. In this way one obtains the topological sphere. At first sight, the sphere seems to have three singular points, or angles, from the $C^{\infty}$ (and the more, from the complex-analytic) point of view. But it is not true, as the quotient $T / \Gamma$ can be 


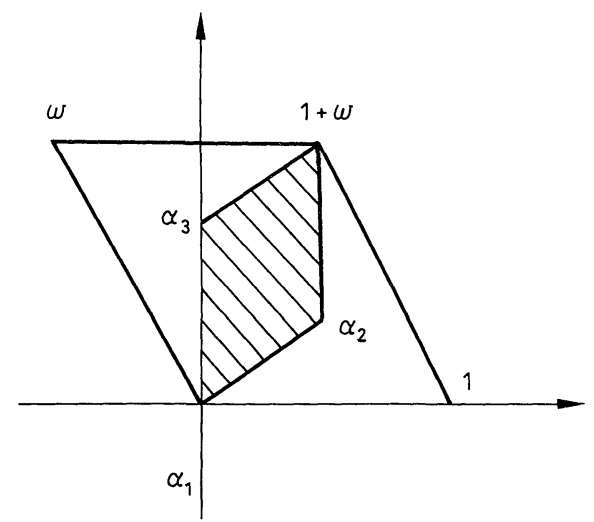

Fig. 1. The Coxeter transformation on the torus of type $\mathscr{A}_{2}$

defined in the complex-analytic category as a smooth Riemann surface by specifying the set of its local coordinates $w_{P}$ in the neighbourhood of each point $P$ :

$$
P=T / \Gamma, \quad w_{P}= \begin{cases}z, & \text { if } P \neq \alpha_{1}, \alpha_{2}, \alpha_{3} \\ \left(z-\alpha_{i}\right)^{3}, & \text { if } P=\alpha_{1}, \alpha_{2}, \text { or } \alpha_{3} .\end{cases}
$$

It is not so in the three-dimensional case, where the quotient $T_{\mathbb{C}}^{3} / \Gamma$ is a singular complex space, and it is necessary to resolve its singularities. Let us return to our construction.

Step 1. The description of all complex structures on $T^{6}$ which are invariant under $\Gamma$. This does not cause any difficulty. There are three complex coordinates $z_{k}$ in $\mathbb{R}^{6}+i \mathbb{R}^{6}$ and the three conjugates $\bar{z}_{k}$, each $z_{k}$ corresponding to the eigenspace of $c$ with the eigenvalue $\lambda_{j_{k}}, k=1,2,3$, where

$$
\lambda_{j}=\exp \frac{2 \pi i}{h} m_{j}, \quad \bar{\lambda}_{j}=\lambda_{7-j},
$$

and $\bar{z}_{k}$ corresponding to the conjugate subspace. The choice of three from the six complex coordinates, say, $z_{1}, z_{2}, z_{3}$, specifies the complex structure on $T^{6}$ and turns $\Gamma$ to a group of holomorphic isometric automorphisms of the torus $T_{\mathbb{C}}^{3}=\mathbb{C}^{3} / L .^{2}$

Step 2. The choice among all possible complex structures of that with respect to which the holomorphic 3-form $d z_{1} \wedge d z_{2} \wedge d z_{3}$ is invariant under $\Gamma$. In that case, it descends to the quotient-space $K_{0}=T_{\mathbb{C}}^{3} / \Gamma$ as a holomorphic 3-form; let us denote the resulting form on $K_{0}$ by $\omega_{0}$. The assumption that such a complex structure exists prohibits quite a number of algebras.

Proposition 1. Let $G$ be a finite group acting on a CYS $M$, and $\omega \in \Gamma\left(\Omega_{M}\right)$ a holomorphic volume element on $M$. Let $x_{0}$ be a fixed point of $G$. Then the action of $G$

\footnotetext{
${ }^{2}$ One could have started from the beginning with complex tori $T_{\mathbb{C}}^{3}$ having non-trivial symmetries. Such tori are completely classified [14]
} 
defines the subgroup $G\left(x_{0}\right) \subset \mathrm{GL}_{\mathbb{C}}\left(T_{x_{0}} M\right)$, where $T_{x_{0}} M$ is the tangent space to $M$ at $x_{0}$. For $\omega$ to be invariant under $G$, it is necessary and sufficient that $G\left(x_{0}\right)$ be a subgroup of $\mathrm{SL}_{\mathbb{C}}\left(T_{x_{0}} M\right)$.

Applying the proposition to $\omega=d z_{1} \wedge d z_{2} \wedge d z_{3}$, we get the condition $m_{j_{1}}+m_{j_{2}}+m_{j_{3}}=h$, where $m_{j}$ are the numbers introduced in formula (1) $0 \leqq m_{j}<h$.

Step 3. Resolving singularities. Let $M$ be a three-dimensional CYS, and $G$ a finite abelian group acting on $M$. Suppose that the fixed point sets of elements of $G$ are of codimension $>1$. Then the union of orbits of all the fixed points of elements of $G$ is the singularity set of the quotient variety $M_{0}=M / G$. Denote it by $S, S=\operatorname{Sing} M_{0}$. By a resolution of singularities one means a surjective holomorphic map $f: \tilde{M}_{0} \rightarrow M_{0}$ with connected, compact fibers, such that $M_{0}$ is a smooth (nonsingular) complex variety and $f: \tilde{M}_{0}-f^{-1}(S) \rightarrow M_{0}-S$ is an isomorphism (see Fig. 2).

The resolution of singularities is not unique. In the case under consideration, the arbitrariness is restricted by the assumption that $\tilde{M}_{0}$ be a CYS. This occurs if the 3-form $\omega_{0}$ on $M_{0}$ having no zeros or poles lifts to a 3 -form on $\tilde{M}_{0}$ which possesses the same property, i.e. which does not acquire zeros or poles along divisors glued in place of singular points. The possibility of such resolution is based on the following proposition.

Proposition 2. Let $\operatorname{dim} M=3, x \in \pi^{-1}(S)$, where $\pi: M \rightarrow M_{0}=M / G$ is the natural projection. Let $G_{x}=\operatorname{Stab}_{G}(x), G(x)=\operatorname{im}\left\{G_{x} \hookrightarrow G L_{\mathbb{C}}\left(T_{x} M\right)\right\}$ and suppose, that $G(x) \subset \mathrm{SL}_{\mathbb{C}}\left(T_{x} M\right)$ for all the points $x \in \pi^{-1}(S)$. Then we have:

1) There exists a CYS $K$ and a map $f: K \rightarrow M_{0}$, which is a desingularization of $M_{0}$. 2) For any point $x \in \pi^{-1}(S)$,

$$
\chi\left(f^{-1} \pi(x)\right)=|G(x)|
$$

where $\chi(\cdot)$ denotes the topological Euler characteristics. 3) If there is a holomorphic 3-form $\omega_{0}$ on $M_{0}$ which has no zeros or poles, then the form $f^{*} \omega$ on $K$ is holomorphic and nowhere vanishing, so the canonical bundle $\Omega_{K}$ is trivial.

For the proof see the Appendix, Sect. 5.

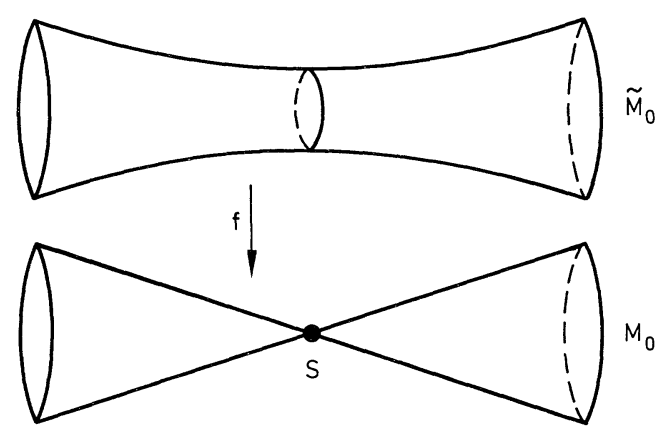

Fig. 2. The resolution of the conic singularity 
Table 1

\begin{tabular}{lllll}
\hline Type of symmetry & Exponents & $N$ & $\chi(K)$ & $h^{11}(K)$ \\
\hline 1. $A_{2}^{(1)} \times A_{2}^{(1)} \times A_{2}^{(1)}$ & $\frac{1}{3}(111)$ & 27 & 72 & 36 \\
2. $A_{2}^{(1)} \times D_{4}^{(1)}, A_{1}^{(1)} \times A_{5}^{(1)}$ & $\frac{1}{6}(123)$ & 12 & 48 & 35 \\
3. $B_{2}^{(1)} \times B_{4}^{(1)}, B_{2}^{(1)} \times D_{4}^{(2)}$ & $\frac{1}{8}(125)$ & 4 & 48 & 27 \\
4. $D_{2}^{(1)} \times D_{4}^{(2)}$ & $\frac{1}{8}(134)$ & 8 & 48 & 28 \\
5. $A_{2}^{(1)} \times D_{4}^{(3)}, A_{2}^{(1)} \times F_{4}^{(1)}, E_{6}^{(1)}$ & $\frac{1}{12}(147)$ & 3 & 48 & 27 \\
6. $A_{2}^{(1)} \times G_{2}^{(1)} \times G_{2}^{(1)}$ & $\frac{1}{6}(114)$ & 3 & 48 & 29 \\
7. $D_{2}^{(1)} \times F_{4}^{(1)}$ & $\frac{1}{12}(156)$ & 4 & 48 & 31 \\
$8 . A_{6}^{(1)}$ & $\frac{1}{7}(124)$ & 7 & 48 & 24 \\
$9 . A_{3}^{(1)} \times A_{3}^{(1)}$ & $\frac{1}{4}(112)$ & 16 & 48 & 31 \\
\hline
\end{tabular}

From Part 1 of Proposition 2 we deduce that if Step 2 is executable for the given group $\Gamma$, then automatically Step 3 is executable, i.e. there exists a resolution of singularities $f: K=\widetilde{K}_{0} \rightarrow K_{0}$ in which $K$ is a CYS.

Step 4. Calculation of invariants of $K$. Formula (2) and the additivity of the Euler characteristic make it possible to evaluate $\chi(K)$ (see Sect. 3). The calculation of the fundamental group is described in Sect. 3; it turns out that $\pi_{1}(K)=0$. In Sect. 4 we calculate the Hodge numbers of the 3. CYS from our table.

Theorem. The following table contains the complete list of CYS that can be obtained with the help of the described procedure.

Explanation of the Table. (i) In the first column, the superscript is equal to the order of an exterior automorphism of the Dynkin diagram, by which the Coxeter element is twisted.

(ii) In the second column, the entry of the form $\frac{1}{h}\left(a_{1}, a_{2}, a_{3}\right)$ shows that the action of $c$ can be written in the form $c:\left(z_{1}, z_{2}, z_{3}\right) \mapsto\left(\varepsilon^{a_{1}} z_{1}, \varepsilon^{a_{2}} z_{2}, \varepsilon^{a_{3}} z_{3}\right), \varepsilon=e^{\frac{2 \pi i}{h}}$ in coordinates $z_{1}, z_{2}, z_{3}$ defining the complex structure on $T^{6}$.

(iii) $N$ is the number of fixed points of $c$, which is, according to the Lefschetz theorem, equal to the value of the characteristic polynomial of the linear map $c \in \operatorname{End}\left(\mathbb{R}^{6}\right)$ at unity. It coincides with the number of fundamental weights of the algebra.

(iv) Another important invariant is the Hodge number $h^{2,1}(K)$. It can be obtained from the table and the formula $h^{1,1}-h^{2,1}=\frac{1}{2} \chi(K)$.

\section{Calculation of Topological Invariants}

\section{Euler Characteristic}

Now we want to prove the Dixon-Harvey-Vafa-Witten formula for the Euler characteristic $\chi(K)$ of a CYS $K$ [9]. This formula reads as follows. Let $G$ be an abelian group acting on a two- or three-dimensional CYS $M$, and suppose that the quotient $M / G$ admits a resolution of singularities with the trivial canonical bundle $\Omega_{K}$. Let us denote by $M(g, h)$ the set of points in $M$ which are fixed under both elements $g$ and $h$ of $G$, and by $\chi(g, h)$ its Euler characteristic. Then

$$
\chi(K)=\frac{1}{|G|} \sum_{g, h \in G} \chi(g, h) .
$$


Lemma 1. Let $x \in M$ and $\operatorname{Stab}_{G}(x)=F$ (the subgroup of all elements of $G$ which fix $x$ ). Then the fiber of the map $K \rightarrow M / G$ (resolving singularities) over the orbit of $x$ has Euler characteristic $|F|$.

Proof. Apply the corollary from Proposition 5 of the Appendix. Let $\pi: M \rightarrow M / G$ and $f: K \rightarrow M / G$ denote the natural maps. The singular point $\pi(x) \in M / G$ is locally equivalent to the quotient of the neighbourhood of $x \in M$ under $F$, and it is, in its turn, isomorphic to the quotient $\mathbb{C}^{n} / F(n=2,3)$ under a linear diagonal action of $F$. Hence we have to prove only that the Euler characteristic of a toric variety is equal to the number of cones of the maximal dimension $n$ in its fan. This follows from the decomposition

$$
X_{\Sigma}=\coprod_{\sigma \in \Sigma} \mathbb{D}_{\sigma}
$$

where $\mathbb{D}_{\sigma} \simeq\left(\mathbb{C}^{*}\right)^{n-\operatorname{dim} \sigma}$, if $n>\operatorname{dim} \sigma$, and $\mathbb{D}_{\sigma}=\{$ point $\}$, if $n=\operatorname{dim} \sigma$ (see Sect. 4 of Appendix). As $\chi\left(\left(\mathbb{C}^{*}\right)^{K}\right)=\chi\left(\mathbb{C}^{*}\right)^{K}=0$, we have

$$
\chi\left(X_{\Sigma}\right)=\sum_{\sigma \in \Sigma} \chi\left(\mathbb{\Phi}_{\sigma}\right)=\sum_{\substack{\sigma \in \Sigma \\ \operatorname{dim} \sigma=n}} \chi(\text { point })=L,
$$

where $L$ is the number of $n$-dimensional cones in $\Sigma$.

For any subgroup $F \subset G$ we put:

$$
\begin{gathered}
M_{F}:=\left\{x \in M: \operatorname{Stab}_{G}(x)=F\right\}, \\
\bar{M}_{F}:=\bigcup_{F \subset F^{\prime}} M_{F^{\prime}}=\left\{x \in M: F \subset \operatorname{Stab}_{G}(x)\right\} .
\end{gathered}
$$

Then $\pi\left(M_{F}\right)=M_{F} /(G / F)$ and

$$
\chi\left(\pi\left(M_{F}\right)\right)=\frac{\chi\left(M_{F}\right)}{[G: F]} .
$$

Since the resolution of singularities blows up points in $\pi\left(M_{F}\right)$ to subvarieties of the Euler characteristic $|F|$, which form a locally trivial bundle over $\pi\left(M_{F}\right)$, we have

$$
\chi\left(f^{-1} \pi\left(M_{F}\right)\right)=|F| \chi\left(\pi\left(M_{F}\right)\right) .
$$

Hence

$$
\chi(K)=\sum_{F \subset G} \frac{|F|}{[G: F]} \chi\left(M_{F}\right)=\frac{1}{|G|} \sum_{F \subset G}|F|^{2} \chi\left(M_{F}\right) .
$$

Observe now that

$$
\chi(g, h)=\chi\left(\prod_{\{g, h\} \subset F} M_{F}\right)=\sum_{\{g, h\} \subset F} \chi\left(M_{F}\right) .
$$

Substituting this into formula (3) and changing the order of summing, we get

$$
\chi(K)=\frac{1}{|G|} \sum_{F \subset G} \sum_{\{g, h\} \subset F} \chi\left(M_{F}\right)=\frac{1}{|G|} \sum_{F \subset G}|F|^{2} \chi\left(M_{F}\right),
$$

which is exactly the same as written above. Thus the formula (3) is proved. 


\section{Number of Fixed Points}

The Euler characteristic of the fixed point set of a given transformation on a manifold $M$ can be evaluated with the help of the Lefschetz formula, which reads as follows:

$$
\chi_{g}=\chi(x \in M: g x=x)=\sum_{i=0}^{m}(-1)^{i} \operatorname{Tr}\left\{g^{*}: H^{i}(X, \mathbb{C}) \rightarrow H^{i}(X, \mathbb{C})\right\}
$$

where $m=\operatorname{dim}_{\mathbb{R}} M$. In the case when $M=T^{m}$ is a torus, we have

$$
H^{1}(M, \mathbb{C}) \cong \mathbb{C}^{m} \cong \mathbb{C} d x_{1}+\ldots+\mathbb{C} d x_{m},
$$

and

$$
H^{p}(X, \mathbb{C})=\Lambda^{p} H^{1}(X, \mathbb{C}) .
$$

We consider the case when the action of $g$ can be written in the form

$$
g:\left(x_{1}, \ldots, x_{m}\right) \mapsto\left(\lambda_{1} x_{1}, \ldots, \lambda_{m} x_{m}\right) .
$$

Then

$$
\chi_{g}=1-\sum_{i} \lambda_{i}+\sum_{i<j} \lambda_{i} \lambda_{j}-\sum_{i<j<k} \lambda_{i} \lambda_{j} \lambda_{k}+\ldots+(-1)^{m} \lambda_{1} \lambda_{2} \ldots \lambda_{m}=P_{g}(1),
$$

where $P_{g}$ is the characteristic polynomial of the linear endomorphism $g \in \operatorname{End}\left(\mathbb{R}^{m}\right)$. The characteristic polynomials of Coxeter elements (see Sect. 2) are known [11].

A slight generalization of the Lefschetz formula makes the calculation of $\chi(K)$ by formula (3) purely algorithmic. This is the following formula for

$$
\chi(g, h)=\frac{1}{|F|} \sum_{i=0}^{m}(-1)^{i} \sum_{s \in F} \operatorname{Tr}\left\{s^{*}: H^{i}(M) \rightarrow H^{i}(M)\right\}=\frac{1}{|F|} \sum_{s \in F} P_{s}(1),
$$

where $F$ denotes the subgroup in $G$ generated by $g, h$, and $s=g^{k} h^{\ell}$ runs over all elements of $F$.

The calculation of the number of fixed curves (in some cases, powers of the Coxeter elements have fixed curves) reduces to the calculation of the number of fixed points for fewer dimensions (one should merely forget about the coordinate changing along a fixed curve).

\section{Fundamental Group}

It follows from Sect. 6 of the Appendix that the resolution of singularities does not change the fundamental group, so it is sufficient to know the fundamental group of the singular quotient-space $K_{0}$.

Turn to the proof of the fact that the varieties $K$ of Theorem of 2 are simply connected. To make it clearer we shall consider the case when $h$ is a prime; then all powers of $c$ have the same fixed set $\left\{P_{1}, \ldots, P_{q}\right\}=S$ in $T^{3}=M$. Let $M_{1}=M-S$, $K_{1}=(M-S) / \Gamma \subset K_{0}=M / \Gamma ; S^{\prime}=\left\{P_{1}^{\prime} \ldots P_{q}^{\prime}\right\}=K_{0}-K_{1}=\pi(S)$, where $\pi: M \rightarrow K_{0}$ is the natural projection. As $\Gamma$ acts freely on $M_{1}, \pi_{1}\left(K_{1}\right)$ is the extension of $\pi_{1}\left(M_{1}\right)$ by $\Gamma$. This means that every element of $\pi_{1}\left(K_{1}\right)$ can be written in the form $\sigma \gamma$ with $\sigma \in \Gamma$ and $\gamma \in \pi_{1}\left(M_{1}\right)$, and we have

$$
\sigma \gamma \sigma^{\prime} \gamma^{\prime}=\sigma \sigma^{\prime} \gamma^{\sigma^{\prime}} \gamma^{\prime} \quad \text { (the group law), }
$$


where $\gamma^{\sigma^{\prime}}$ denotes the image of $\gamma$ under the transformation $\sigma^{\prime}$. In particular $\gamma^{\sigma}=\sigma^{-1} \gamma \sigma$. Thus the lattice $\pi_{1}\left(M_{1}\right) \cong \mathbb{Z}^{6}$ is a normal subgroup in $\pi_{1}\left(K_{1}\right)$ of index $h=|\Gamma|$.

Consider the set of punctured neighbourhoods $U_{1}, \ldots, U_{q}$ of points $P_{1}^{\prime}, \ldots, P_{q}^{\prime}$; by Sect. 2 they are local-analytically equivalent to the toric variety $X_{\left(\mathbb{R}^{n}, N, \Sigma\right)}$, where the fan $\Sigma$ contains all proper faces of the cone $\sigma=\mathbb{R}_{+}^{n}$ (and does not contain $\sigma$ itself). By Lemma 3(i), $N_{\Sigma}$ is the lattice generated by $\kappa_{1}, \kappa_{2}, \kappa_{3}$, that is $N_{\Sigma}=N_{0}$, and $N / N_{\Sigma} \cong \Gamma$ (if $h$ is not a prime, then it may turn out that $\kappa_{1}, \kappa_{2}, \kappa_{3}$ do not generate all of $N_{\Sigma}$, since the sides of the triangle $H \cap \sigma$ may contain points of $N$ other than $\kappa_{j}$ ). So the local fundamental groups $\pi_{1}\left(U_{j}\right)$ are isomorphic to $\Gamma$ and are generated by one loop "surrounding" $P_{j}^{\prime}$, which will be denoted by $\sigma_{j}$. We have $\sigma_{j}^{h}=1\left[\right.$ in $\left.\pi_{1}\left(U_{j}\right)\right]$. If we choose the distinguished point $x_{0}$ to refer elements of $\pi_{1}$ in $U_{1}$, we shall get the following set of generators for $\pi_{1}\left(K_{1}\right)$ :

$$
\sigma=\tilde{\sigma}_{1}=\sigma_{1}, \quad \tilde{\sigma}_{j}=\sigma_{1 j}^{-1} \sigma_{j} \sigma_{1 j} \quad(j=2, \ldots, q),
$$

where $\sigma_{1 j}$ is the segment of the straight line leading from $x_{0}$ to $U_{j}$. Then $\tilde{\sigma}_{j}=\sigma \gamma_{j}$, where $\left\{\gamma_{j}\right\}$ is the set of representatives of cosets in $\mathbb{Z}^{6} /(\sigma-I) \mathbb{Z}^{6}$ (the proof is straightforward). Observe that the elements $\left\{\tilde{\sigma}_{j}\right\}$ yield a complete system of representatives of conjugacy classes in $\pi_{1}\left(K_{1}\right)$. Indeed, one can easily deduce from definitions the following fact: if the elements $\sigma \gamma^{\prime}$ and $\sigma \gamma^{\prime \prime}$ are conjugated by an element $\sigma_{1} \gamma$, then $\gamma^{\prime \prime}=\gamma^{\prime}\left(\gamma^{\prime \sigma_{1}} \gamma^{\prime-1}\right) \cdot\left(\gamma^{\sigma} \gamma^{-1}\right)^{-1}$. Taking additive notation, we get

$$
\gamma^{\prime \prime}-\gamma^{\prime}=\left(\sigma_{1}-I\right) \gamma^{\prime}-(\sigma-I) \gamma
$$

Note now that $\sigma_{1}=\sigma^{k}$, and

$$
\begin{aligned}
\left(\sigma_{1}-I\right) \gamma^{\prime} & =\sigma^{k} \gamma^{\prime}-\gamma^{\prime}=\sigma^{k} \gamma^{\prime}-\sigma^{k-1} \gamma^{\prime}+\sigma^{k-1} \gamma^{\prime}-\sigma^{k-2} \gamma^{\prime}+\ldots \\
& =(\sigma-I)\left(\sigma^{k-1} \gamma^{\prime}+\ldots+\sigma \gamma^{\prime}+\gamma^{\prime}\right)=(\sigma-I) \tilde{\gamma} .
\end{aligned}
$$

From (4), it follows that pasting holes in $U_{j}$ kills the generators $\sigma_{j}$ of the fundamental group (the loop $\sigma_{j}$ can be contracted to a point, and after that $\tilde{\sigma}_{j}$ can be contracted along the segment $\sigma_{i j}$ ). Thus $K_{0}$ is simply connected (and hence, so is $K$ ).

\section{Calculation of Yukawa Couplings}

It has been shown in [6] that triple couplings between fermionic massless $27-E_{6}$ multiplets can be calculated by purely topological methods; they are merely intersection indices $\left(F_{j} \cdot F_{i} \cdot F_{k}\right)$ of triplets of cohomology classes in $H^{1,1}(K, \mathbb{C})$. In this section, we treat one example in detail and explain the method for calculating the group $H^{1,1}(K, \mathbb{C})$ and the trilinear intersection form on it. We shall identify elements of $H^{1,1}(K, \mathbb{C})$ with the corresponding sums of 4-surfaces in $K$.

Let $\pi: T \rightarrow K_{0}=T / \Gamma$ be the quotient map, and $f: K \rightarrow K_{0}$ that of desingularization. Then

$$
H^{2}(K, \mathbb{C})=H^{2}(T, \mathbb{C})^{\Gamma} \oplus \Sigma \mathbb{C} E_{j},
$$

where $\left\{E_{j}\right\}$ is the set of all exceptional divisors of the resolution of singularities [i.e. all the divisors on $K$ for which $f(E)$ is not a divisor on $\left.K_{0}\right]$. As $H^{2,0}(K, \mathbb{C})$ 
$=H^{0,2}(K, \mathbb{C})=0$ (there are no holomorphic or antiholomorphic 2-forms on $T$ ), one can write $H^{1,1}$ instead of $H^{2}$ in (5).

We shall illustrate the calculations for one example, namely $B_{2}^{(1)} \times B_{4}^{(1)}$. The action of $c$ has the form

$$
\left(z_{1}, z_{2}, z_{3}\right) \mapsto\left(\varepsilon z_{1}, \varepsilon^{2} z_{2}, \varepsilon^{5} z_{3}\right), \quad \varepsilon=e^{\frac{2 \pi i}{8}} .
$$

Here $H^{1,1}(T, \mathbb{C})^{\Gamma}$ is generated by three elements $d z_{i} \wedge d \bar{z}_{i}, i=1,2,3$, so $\operatorname{dim} H^{1,1}(T, \mathbb{C})^{T}=3$. To observe all the divisors $E_{j}$, we decompose the transformation $T \rightarrow K$ into the chain of three similar transformations, each one including taking quotient under $\mathbb{Z}_{2}$ (the first $\mathbb{Z}_{2}$ is $\left\{1, c^{4}\right\}$, the second one is $\left\{1, c^{2} \bmod c^{4}\right\}$ and the third one is $\left.\left\{1, c \bmod c^{2}\right\}\right)$ :

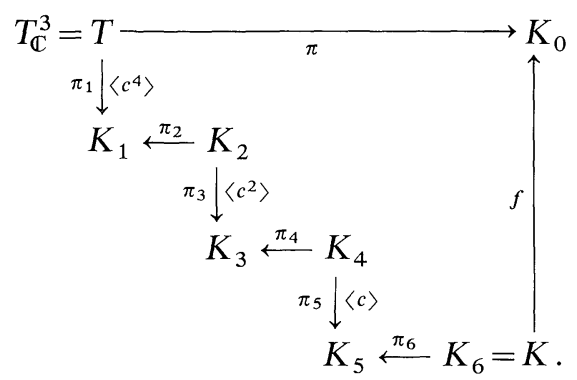

Here $\pi_{1}, \pi_{2}, \pi_{3}$ denote the quotient-maps and $\pi_{2}, \pi_{4}, \pi_{6}$ denote the resolutions of singularities.

Step 1. $T / \mathbb{Z}_{2}=K_{1}, \mathbb{Z}_{2}=\left\{1, c^{4}\right\}, c^{4}=\frac{1}{8}(4,8,20)=\frac{1}{2}(1,0,1)$. There are 16 fixed curves $\left\{z_{1}=\omega_{j}, z_{3}=\omega_{k}\right\}, i, j=1, \ldots, 4$. Replacing $z_{1}-\omega_{j}$ and $z_{3}-\omega_{k}$ by $z_{1}$ and $z_{3}$ respectively, we get the local description of the resolution of singularities in the neighbourhood of these points

$$
\begin{aligned}
& \left(z_{1}, z_{2}, z_{3}\right) \stackrel{\text { quotient map }}{\longrightarrow}\left(z_{1}^{2}, z_{1} z_{3}, z_{3}^{2}, z_{2}\right) \\
& x \quad y \quad z \\
& y^{2}=x z .
\end{aligned}
$$

So we get the quadratic singular point. The desingularization is covered by two coordinate maps:

$$
\begin{aligned}
& U_{1}:\left(x, \frac{y}{x}, z_{2}\right)=\left(z_{1}^{2}, \frac{z_{3}}{z_{1}}, z_{2}\right), \\
& U_{2}:\left(z, \frac{y}{z}, z_{2}\right)=\left(z_{3}^{2}, \frac{z_{1}}{z_{3}}, z_{2}\right) .
\end{aligned}
$$

Over each point of the complex $z_{2}$-curve,

$$
z_{1}^{2}=0, z_{2}=\text { const }\left(\text { in } U_{1}\right) \text { or } z_{3}^{2}=0, z_{2}=\text { const }\left(\text { in } U_{2}\right),
$$

there is a complex curve covered by two coordinate maps: $u=z_{3} / z_{1}$ and $1 / u$. Such a curve is isomorphic to $\mathbb{C P}^{1}$. Thus the resolution of singularities glues in 16 divisors of the form $B_{j} \times \mathbb{C P}^{1}$ in place of 16 singular elliptic $z_{2}$-curves $B_{j}(j=1, \ldots, 16)$. 
Step 2. $K_{3}=K_{2} / \mathbb{Z}_{2}, \mathbb{Z}_{2}=\left\{1, c^{2}\right\}, c^{2}=\frac{1}{4}(1,2,1)$. The element $c^{2}$ has 16 fixed points in $T_{\mathbb{C}}^{3}$,

$$
z_{1}=z_{3}=\omega_{j}, \quad z_{2}=\omega_{k} \quad(j, k=1, \ldots, 4)
$$

which lie on four of sixteen curves defined in Step 1. On $K_{2}$ the fixed points of $c^{2}$ lie over these sixteen points, or on sixteen curves $\mathbb{C P}^{1}$ over them. In correspondence with (6), we have:

$$
\begin{aligned}
& U_{1}: \quad c^{2}:\left(z_{1}^{2}, \frac{z_{3}}{z_{1}}, z_{2}\right) \mapsto\left(-z_{1}^{2}, \frac{z_{3}}{z_{1}},-z_{2}\right), \\
& U_{2}: \quad c^{2}:\left(z_{3}^{2}, \frac{z_{1}}{z_{3}}, z_{2}\right) \mapsto\left(-z_{3}^{2}, \frac{z_{1}}{z_{3}},-z_{2}\right) .
\end{aligned}
$$

We see that $\mathbb{C P}^{1}$ is fixed under $c^{2}$. The resolution is covered by four maps $U_{11}, U_{12}, U_{21}, U_{22}$. For example, we have

$$
\begin{aligned}
& U_{11}:\left(z_{1}^{4}, \frac{z_{2}}{z_{1}^{2}}, \frac{z_{3}}{z_{1}}\right), \\
& U_{12}:\left(z_{2}^{2}, \frac{z_{1}^{2}}{z_{2}}, \frac{z_{3}}{z_{1}}\right) .
\end{aligned}
$$

Again we have $\mathbb{C P}^{1}$ glued in over each point of the old $\mathbb{C} \mathbb{P}^{1}$, with two coordinates $u=\frac{z_{2}}{z_{1}^{2}}$ and $u=\frac{z_{1}^{2}}{z_{2}}=\frac{1}{u}$. We shall call the four divisors glued in over fixed points of $c^{2}$ divisors of type $(*)$. Those 12 exceptional $B_{j} \times \mathbb{C P}^{1}$ which do not contain fixed points of $c^{2}$ are permuted by $c^{2}$ in orbits of length 2 , so after applying the quotient map they give 6 divisors isomorphic to $B \times \mathbb{C P}^{1}$ (see Fig. 3).

Step 3. $K_{5}=K_{4} / \mathbb{Z}_{2}, \mathbb{Z}_{2}=\{1, c\}, c=\frac{1}{8}(1,2,5)$. The element $c$ has 4 fixed points,

$$
z_{2}=\omega_{j}, \quad z_{1}=z_{3}=\omega_{k}, \quad j, k=1,3 .
$$
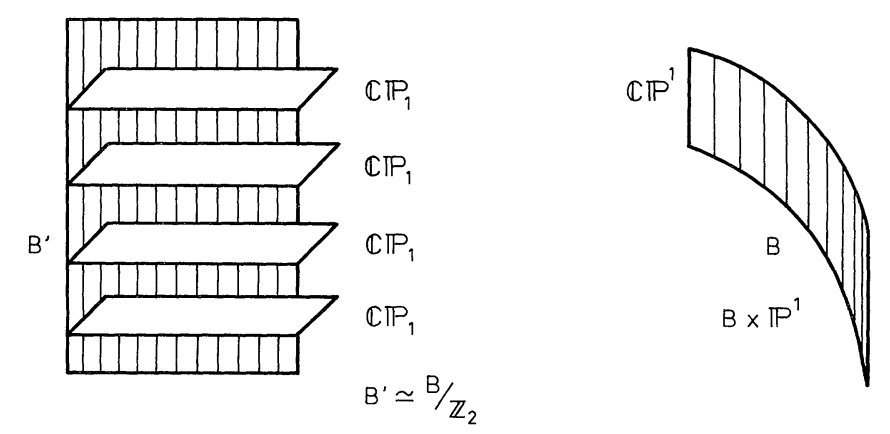

a

Fig. 3. a The structure of an exceptional divisor of type (*). b The structure of an exceptional divisor of type $B \times \mathbb{P}^{1}$ 
The six divisors $B \times \mathbb{C P}^{1}$ are permuted in orbits by two, two of the four divisors of type $(*)$ are also permuted so there are 3 divisors $B \times \mathbb{C P}^{1}, 1$ divisor of type $(*)$ and two divisors of the more complicated form in $K_{6}=K$. The last two divisors will be said to be of type (**). They are obtained by applying the quotient map to a divisor of type $(*)$ under $\mathbb{Z}_{2}$ and desingularizing it. The action of $c$ permutes two ruled surfaces which arose at Step 2 [we now consider only one of the two divisors of type (*)], and the other two are glued to itself. In coordinates introduced in (7), we have:

$$
\begin{aligned}
& U_{11}: \quad c:\left(z_{1}^{4}, \frac{z_{2}}{z_{1}^{2}}, \frac{z_{3}}{z_{1}}\right) \mapsto\left(-z_{1}^{4}, \frac{z_{2}}{z_{1}^{2}},-\frac{z_{3}}{z_{1}}\right), \\
& U_{12}: \quad c:\left(z_{2}^{2}, \frac{z_{1}^{2}}{z_{2}}, \frac{z_{3}}{z_{1}}\right) \mapsto\left(-z_{2}^{2}, \frac{z_{1}^{2}}{z_{2}},-\frac{z_{3}}{z_{1}}\right) .
\end{aligned}
$$

We see, that the fixed set is the $z_{2} / z_{1}^{2}$-curve which was glued in at Step 2 (it has an equation, say, in $U_{11}$, of the form $z_{2}^{4}=z_{3} / z_{1}=0$ ). Similarly, we find also the fixed curve $z_{1}^{4}=z_{1} / z_{3}=0$ in $U_{21}$ or $U_{22}$. The resolution of singularities glues in $\mathbb{C P}^{1}$ over each point of the fixed curves. We get the following divisor (Fig. 4).

We introduce the following notation for components of exceptional divisors: $E_{1}, E_{2}, E_{3}$ are divisors of type $B \times \mathbb{C P}^{1} ; F$ is the "base" of the divisor of type (*); $F_{1}, F_{2}, F_{3}, F_{4}$ are the remaining components of the divisor of type (*); $G^{k}$ is the "base" of the divisor of type (**); $G_{0}^{k}, G_{1}^{k}, G_{2}^{k}, G_{i j}^{k}(i, j, k=1,2)$ are defined by the picture. We see that there are 24 exceptional divisors, so

$$
h^{1,1}(K)=\operatorname{dim} H^{11}(T)^{\Gamma}+24=27 .
$$

There are three more generators of $H^{1,1}(K)$; we shall choose for them the following 4-surfaces: 1) $S$, the image of the torus $z_{2}=C$ (for a generic $C$ ) in $K$; $S$ is a $K 3$ surface having Picard number 20 ;2) $S_{1}$, the image of the torus $z_{3}=C$; 3) $S_{2}$, the image of the torus $z_{1}=C$. It is immediate to verify that many Yukawa constants are zero, because the corresponding 4-surfaces do not intersect. For example:

$$
\begin{gathered}
\left(E_{i} \cdot E_{j} \cdot D\right)=\left(F_{k} \cdot F_{\ell} \cdot D\right)=0 \quad(i \neq j, k \neq \ell) \\
\left(E_{i} \cdot G_{\alpha}^{k} \cdot D\right)=\left(F_{\ell} \cdot G_{\alpha}^{k} \cdot D\right)=0 \\
(k=1,2 ; \alpha=\emptyset, 0,1,2,(11),(12),(21) \cdot(22)) \\
\left(G_{0}^{k} \cdot G_{i}^{k} \cdot D\right)=\left(G_{0}^{k} \cdot G_{i j}^{k} \cdot D\right)=0 ; \\
\left(G_{\alpha}^{1} \cdot G_{\beta}^{2} \cdot D\right)=\left(G_{\kappa}^{k} \cdot S_{i} \cdot D\right)=0 \\
(\alpha, \beta, \kappa \text { are multi-indices, } \kappa \neq \emptyset) ; \\
\left(S^{2} \cdot D\right)=\left(S_{i} \cdot F_{\ell} \cdot D\right)=\left(S \cdot F_{\ell} \cdot D\right)=\left(S_{i} \cdot G_{\kappa}^{k} \cdot D\right)=\left(S \cdot G_{\kappa}^{k} \cdot D\right)=0 \\
(\kappa \neq \emptyset ; k=1,2)
\end{gathered}
$$

and so on. Here $D$ is any one of the 27 basic divisors. More thorough geometrical consideration yields the following indices:

$$
\begin{array}{cc}
\left(S_{i}^{2} \cdot S\right)=4 ; \quad\left(S_{1} \cdot S_{2} \cdot S\right)=8 ; & \left(E_{i}^{2} \cdot S\right)=-8 ; \\
\left(G^{k} \cdot G^{k} \cdot S\right)=-2 ; \quad\left(F^{2} \cdot S\right)=-4 ; & \left(G_{i}^{k} \cdot G^{k} \cdot G_{i j}^{k}\right)=1,
\end{array}
$$




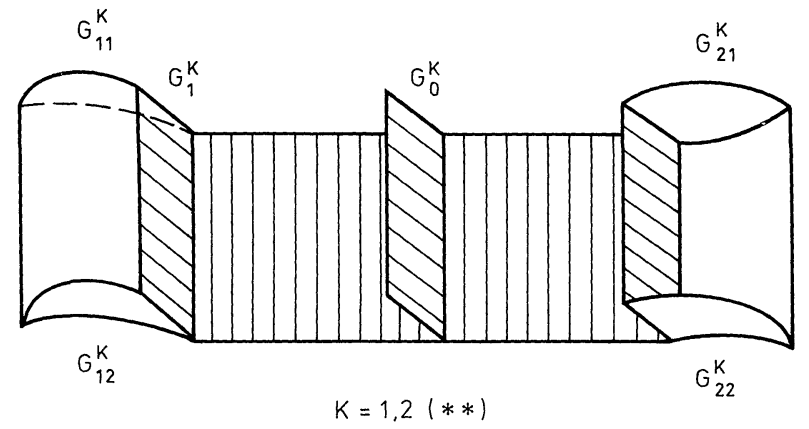

Fig. 4. The structure of an exceptional divisor of type (**)

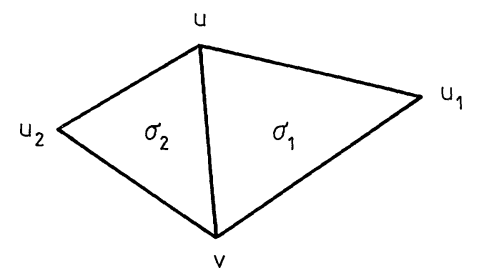

Fig. 5. $u, v, u_{1}, u_{2}$ are primitive vectors of the lattice $N$ (see the Appendix) lying on the edges of cones from the fan $\Sigma$. To each such vector there corresponds a divisor on the toric variety $X$. The segment joining $u$ and $v$ corresponds to the curve which is an interaction of divisors $D_{u} \cap D_{v}$. The triangle spanned by three vectors, say $u, v$, and $u_{1}$, corresponds to the point of the triple interaction $D_{u} \cap D_{v} \cap D_{u_{1}}$

and so on. To calculate indices containing $\left(G^{k}\right)^{2}$ or $F^{2}$, one must write out explicitly the normal bundles of $F$ and $G^{k}$. Some cubes can be easily calculated. For example,

$$
\left(E_{i}^{3}\right)=\left(E_{i} \cdot E_{i} \cdot E_{i}\right)=0 \text {. }
$$

The method for calculating intersection indices of the form $\left(D_{1}^{2} \cdot D\right)$ and $\left(D^{3}\right)$ with $D_{1} \cap D_{2}, D_{1}$ or $D_{2}$ (respectively $D$ ) projecting to a point in $K_{0}$ is given by toric geometry.

Remind that to each primitive vector $u$ lying on a one-dimensional edge of the fan $\Sigma$ (see Sect. 4 of the Appendix) one can associate the divisor $D_{u}$. One has $\left(D_{u} \cdot D_{v} \cdot D_{w}\right)=1$ if the cone $\langle u, v, w\rangle$ belongs to $\Sigma$. Further, if there is a subfan in $\Sigma$ of the form depicted in Fig. 5, then

$$
u_{1}+u_{2}+a u+b v=0
$$

for some $a, b \in \mathbb{Z}$ and

$$
\left(D_{u}^{2} \cdot D_{v}\right)=a, \quad\left(D_{u} \cdot D_{v}^{2}\right)=b,
$$

To calculate $\left(D_{u}^{3}\right)$, one must replace it by $\left(D_{u}^{2} \cdot\left(D_{u}+(\varphi)\right)\right)$, where $\varphi$ is an appropriate rational function, $(\varphi)$ is its divisor. One should choose $\varphi$ in such a manner that the divisor $D_{u}+(\varphi)$ does not contain $D_{u}$ as its component. In toric geometry, it is 
always possible to find a monomial $x^{m}$ with such property. We have

$$
\left(x^{m}\right)=\sum_{\mathbb{R}+u_{i} \in \Sigma}\left\langle u_{i}, m\right\rangle D_{u_{i}} .
$$

Let us turn to Example 4 from Sect. 4 of Appendix. We have

$$
\begin{array}{ll}
\left(D_{\alpha_{1}}^{2} \cdot D_{\alpha_{2}}\right)=\left(D_{\alpha_{1}} \cdot D_{\alpha_{2}}^{2}\right)=-1 ; & \left(D_{\alpha_{3}}^{2} \cdot D_{\alpha_{4}}\right)=\left(D_{\alpha_{3}} \cdot D_{\alpha_{4}}^{2}\right)=0 ; \\
\left(D_{\alpha_{1}}^{2} \cdot D_{\alpha_{3}}\right)=\left(D_{\alpha_{1}}^{2} \cdot D_{\alpha_{4}}\right)=-2 ; & \left(D_{\alpha_{1}} \cdot D_{\alpha_{3}}^{2}\right)=\left(D_{\alpha_{1}} \cdot D_{\alpha_{4}}^{2}\right)=0 .
\end{array}
$$

For calculating $D_{\alpha_{1}}^{3}$, we use the relation

$$
D_{\alpha_{1}}=-2 D_{\kappa_{2}}+D_{\kappa_{3}}-D_{\kappa_{1}}-D_{\alpha_{4}}+\left(x^{m}\right),
$$

where $m$ is defined by its values on the base of the dual lattice: $\left\langle\alpha_{1}, m\right\rangle=1,\left\langle\alpha_{2}, m\right\rangle$ $=\left\langle\alpha_{3}, m\right\rangle=0$.

We need also indices

$$
\left(D_{\alpha_{1}}^{2} \cdot D_{\kappa_{2}}\right)=-3, \quad\left(D_{\alpha_{1}}^{2} \cdot D_{\alpha_{4}}\right)=-2, \quad\left(D_{\alpha_{1}}^{2} \cdot D_{\kappa_{3}}\right)=\left(D_{\alpha_{1}}^{2} \cdot D_{\kappa_{1}}\right)=0 .
$$

Thus we find out:

$$
D_{\alpha_{1}}^{3}=-2\left(D_{\alpha_{1}}^{2} \cdot D_{\kappa_{2}}\right)+\left(D_{\alpha_{1}}^{2} \cdot D_{\kappa_{3}}\right)-\left(D_{\alpha_{1}}^{2} \cdot D_{\kappa_{1}}\right)-\left(D_{\alpha_{1}}^{2} \cdot D_{\alpha_{4}}\right)=8 .
$$

In terms of the base of $H^{1,1}(K)$ introduced above, we can rewrite (8) and (9) as follows:

$$
\begin{gathered}
\left(\left(G_{i}^{k}\right)^{2} \cdot G^{k}\right)=\left(G_{i}^{k} \cdot\left(G^{k}\right)^{2}\right)=-1 ; \\
\left(\left(G_{i_{1}}^{k}\right)^{2} \cdot G_{i_{2}}^{k}\right)=\left(G_{i_{1}}^{k} \cdot\left(G_{i_{2}}^{k}\right)^{2}\right)=0 ; \\
\left(\left(G_{i}^{k}\right)^{2} \cdot G_{i j}^{k}\right)=-2 ; \quad\left(G_{i}^{k} \cdot\left(G_{i j}^{k}\right)^{2}\right)=0 ; \quad\left(G_{i}^{k}\right)^{3}=8 .
\end{gathered}
$$

The remaining indices are calculated similarly.

\section{Conclusion}

As we have already noted, the table of Sect. 2 does not exhaust all the CYS constructed of complex tori by means of Coxeter automorphism. There is a large class of complex tori admitting non-trivial discrete symmetries [14]. For these tori one can also define the Coxeter transformation and apply the described procedure.

On the other hand, the CYS constructed in Sect. 2 have some residual symmetries, belonging to the group generated by translations by fundamental weights of the corresponding Lie algebra. This group is isomorphic to the center of the Lie algebra. One can take quotients under the action of appropriate subgroups.

Some of our CYS have non-zero $H^{1}($ End $T)$. This provides the possibility to deform the complex structure of the tangent bundle $T K$ (while keeping the complex structure on $K$ fixed). Speaking more precisely, the second, the fourth, the seventh, and the ninth strings of the table give CYS with non-zero $H^{1}($ End $T)$. This easily follows from the fact that the elements of $H^{1}(\operatorname{End} T)$ are harmonic tensor fields of the form $\mathscr{A}_{b \bar{c}}^{a}$ so, to show that $H^{1}(\operatorname{End} T)$ is non-zero, it is enough to find such $\Gamma$-invariant fields on the torus. The desingularization can add some 
new such fields. One can use deformations of the complex structure on $T K$ to obtain new vacuum states. One can also take an arbitrary stable vector bundle $E$ instead of $T K$, having $\mathrm{SU}(3)$ [and even $\mathrm{SU}(4), \mathrm{SU}(5)$ ] as its structure group and satisfying the single condition $c_{2}(E)=c_{2}(K)$ (see [4] for more details). In that case, the number of generations depends on the third Chern class of $E$, but not on the Euler characteristic of $K$.

All the harmonic tensor fields of the form $\mathscr{A}_{a \bar{c}}^{b}$ give rise to superfields on the CYS which are $E_{6}$-singlets [4]. The existence of such superfields makes it possible to give a mass to the right neutrino, while keeping the left one massless. One can also use the non-triviality of $H^{1}($ End $T)$ to break deeply $E_{6}$ preserving supersymmetry, as we know is important from phenomenology.

\section{Appendix. Resolution of Singularities (Toric Method) by D. G. Markushevich}

\section{Generalities}

Toric geometry is the field of algebraic geometry which reduced many algebraic geometrical problems (for example, such as the construction of a resolution of singularities, calculation of intersection indices, mixed Hodge structures, fundamental groups and other invariants) to purely combinatorial questions on lattices and convex polyhedral cones in $\mathbb{R}^{n}$. Toric geometry is concerned with a relatively small class of algebraic varieties, the so-called toric varieties. Suffice it to say that all of them are rational. A variety $X^{n}$ (all varieties here are complex ones) is said to be rational, if there is a dense open subset $U \subset X^{n}$, and $n$ meromorphic functions $f_{1}, \ldots, f_{n}$ on $X^{n}$ regular on $U$, such that the $\operatorname{map}\left(f_{1}, \ldots, f_{n}\right): U \rightarrow \mathbb{C}^{n}$ is an embedding (an equivalent definition: the field of meromorphic functions on $X^{n}$ is isomorphic to the purely transcendental field $\mathbb{C}\left[f_{1}, \ldots, f_{n}\right]$ over $\mathbb{C}$ ). Among curves, only the curve of genus 0 , or the Riemann sphere $\mathbb{C P}^{1}$, is rational. However, in spite of the fact that the class of toric varieties is very small, they serve the fundamental testing ground for conjectures, theorems and proofs concerning more general varieties. Moreover, they give an effective tool to investigate the local structure of singular varieties. Toric method can be applied to build a resolution of singularities of a complete intersection variety (we will not dwell on this construction, see for it [16]) and of a quotient under a finite abelian group. For toric varieties see [15].

As general algebraic varieties are obtained by gluing together affine algebraic varieties, playing the role of coordinate charts, the general toric varieties are obtained by gluing together affine toric varieties. An affine variety is a variety whose points are in a 1-to-1 correspondence with maximal ideals of its function ring. To each point $P$ of a variety $X$ there corresponds the maximal ideal $m$ of the function algebra $A=\mathbb{C}[X]$ consisting of regular (polynomial) functions on $X$ vanishing at $P$. The variety $X$ is said to be affine if the map $P \mapsto m$ from $X$ to the set of maximal ideals of $A$ is bijective.

Let $A$ be any commutative finitely generated algebra with unity over $\mathbb{C}$ without nilpotents. Then the set of maximal ideals of $A$ is an affine algebraic variety. One uses the notation

$$
X=\operatorname{Spec} A \text {. }
$$


An affine variety can be represented as a closed subvariety of some affine space $\mathbb{C}^{N}$. To see this, we choose a set $u_{1}, \ldots, u_{N}$ of generators of $A$ and write down all the relations between them; let

$$
F_{1}\left(u_{1}, \ldots, u_{N}\right)=0, \ldots, F_{t}\left(u_{1}, \ldots, u_{N}\right)=0
$$

be a complete set of the relations. Then $A$ is isomorphic to the quotient of the ring of polynomials by the ideal generated by $F_{1}, \ldots, F_{t}$,

$$
A \simeq \mathbb{C}\left[u_{1}, \ldots, u_{N}\right] /\left(F_{1}\left(u_{1}, \ldots, u_{N}\right), \ldots, F_{t}\left(u_{1}, \ldots, u_{N}\right)\right),
$$

and $X$ is naturally identified with the common set of zeros of $F_{i}$ :

$$
X=\left\{\left(z_{1}, \ldots, z_{N}\right) \in \mathbb{C}^{N} \mid F_{i}\left(z_{1}, \ldots, z_{n}\right)=0, i=1, \ldots, t\right\} .
$$

To obtain the definition of an affine toric variety one must specify that $A$ in (1) is a semigroup algebra. Recall that by the semigroup algebra associated with a semigroup $H$, one means the algebra generated by symbols $\left\{x^{h}\right\}_{h \in H}$, whose products are defined by $x^{h_{1}} \cdot x^{h_{2}}=x^{h_{1}+h_{2}}$ (as we consider only commutative semigroups, we write the semigroup law additively). Any finitely generated semigroup without torsion is isomorphic to a semigroup of the form $\tau \cap \mathbb{Z}^{n}$, where $\tau$ is a convex rational polyhedral cone in $\mathbb{R}^{n}$. The rationality condition means that any face $\tau$ of codimension $k$ spans the linear subspace in $\mathbb{R}^{n}$ that is given by $k$ linear equations with integer coefficients. This condition implies that the semigroup $\tau \cap \mathbb{Z}^{n}$ is finitely generated. One can think of the semigroup algebra $\mathbb{C}\left[\tau \cap \mathbb{Z}^{n}\right]$ as of the algebra of Laurent polynomials generated by monomials $T^{m}=T_{1}^{m_{1}} \ldots T_{n}^{m_{n}}$ whose exponents $m=\left(m_{1}, \ldots, m_{n}\right) \in \tau \cap \mathbb{Z}^{n}$. Of course, the set of exponents $m$ depends on the choice of a basis in the lattice $\mathbb{Z}^{n}$. To eliminate this dependence, we shall give a more abstract definition.

Let us consider a triple $\left(V=\mathbb{R}^{n}, M, \tau\right)$, where $M$ is a complete lattice in $V, \tau$ a convex rational (with respect to $M$ ) polyhedral cone in $V$ with the origin as its vertex. Consider the variety

$$
X^{(V, M, \tau)}=X^{\tau}=\operatorname{Spec} \mathbb{C}[\tau \cap M] .
$$

The algebra of regular functions on $X^{\tau}$ is the semigroup algebra $\mathbb{C}[\tau \cap M]$. One can represent $X^{\tau}$ as a closed algebraic subvariety in some $\mathbb{C}^{N}$ as follows. Let $m^{(1)}, \ldots, m^{(N)}$ be a set of generators of the semigroup $\tau \cap M, u_{1}=T^{m^{(1)}}, \ldots, u_{N}=T^{m^{(N)}}$ a set of generators of $\mathbb{C}[\tau \cap M]$, and let

$$
\left\{F_{i}\left(u_{1}, \ldots, u_{N}\right)=0\right\}_{i=1, \ldots, t}
$$

be the complete set of relations between $u_{i}$. Then $X^{\tau} \subset \mathbb{C}^{N}$, and

$$
X^{\tau}=\left\{\left(z_{1}, \ldots, z_{N}\right) \in \mathbb{C}^{N}: F_{i}\left(z_{1}, \ldots, z_{n}\right)=0, i=1, \ldots, t\right\} .
$$

In addition to (2), we can give explicitly the form of the equations $F_{i}=0$ defining toric varieties. Indeed, all the relations between $u_{j}$ are determined by the relations between $m^{(j)}$. They are linear and have the form

$$
\begin{aligned}
& a_{11} m^{(1)}+\ldots+a_{1 N} m^{(N)}=0 \\
& a_{t 1} m^{(1)}+\ldots+a_{t N} m^{(N)}=0
\end{aligned} \quad\left(a_{i j} \in \mathbb{Z}\right),
$$


so $F_{i}$ have the form

$$
F_{i}\left(u_{1}, \ldots, u_{N}\right)=u_{i_{1}}^{a_{i i_{1}}} \ldots u_{i_{r}}^{a_{i t_{r}}}-u_{j_{1}}^{-a_{l_{j_{1}}}} \ldots u_{N-r^{-r}}^{-a_{i j}} .
$$

We have divided the set of indices $(1, \ldots, N)$ into two parts: $\left(i_{1}, \ldots, i_{r}\right)$ is the set of indices for which $a_{i j} \geqq 0$, and $\left(j_{1}, \ldots, j_{N-r}\right)$ is that for which $a_{i j}<0$. Relations of the form (6) are said to be monomial. For a subvariety of $\mathbb{C}^{N}$ to be toric, it is necessary and sufficient that it be defined by a set of monomial equations. For example, the equations of the two- and three-dimensional quadratic cones admit monomial representations

$$
\begin{array}{ll}
z_{1} z_{2}=z_{3}^{2} & (n=2), \\
z_{1} z_{2}=z_{3} z_{4} & (n=3),
\end{array}
$$

but $n$-dimensional cones with $n>3$ have no monomial equations, so the $n$ dimensional quadratic cone is a toric variety if and only if $n=2$ or 3 .

\section{Properties of Affine Toric Varieties}

Proposition 1. (i) Varieties $X^{\tau}$ are non-singular in codimension 1, that is codim $\operatorname{Sing} X^{\tau} \geqq 2$.

(ii) $X^{\tau}$ is non-singular if $\tau$ is a basic cone, that is $\tau=\mathbb{R}_{+} e_{1}+\ldots+\mathbb{R}_{+} e_{n}$, where $\left(e_{1}, \ldots, e_{n}\right)$ is a basis of the lattice $M$.

(iii) Regular holomorphic exterior forms of the highest degree on $X^{\tau}$ (more precisely, on the non-singular set of $X^{\tau}$ ) are finite linear combinations of the exterior forms of the form $x^{m} \cdot \omega$, where $m$ runs over $M \cap i$, $i$ is the interior of $\tau$, $\omega_{0}=\frac{d x_{1}}{x_{1}} \wedge \ldots \wedge \frac{d x_{n}}{x_{n}}$. In other words,

$$
\Omega_{\tau}:=\Gamma\left(\Omega_{X^{\tau}}\right)=\mathbb{C}[i \cap M] \cdot \omega_{0} .
$$

Proof. For (i) and (iii) see [15]. The second statement is more or less obvious: for $X^{\tau}$ to be smooth, it is necessary that the monomials $u_{1}, \ldots, u_{N}$ defined in Part 1 give a complete system of local parameters for $X^{\tau}$ without relations between them, so $m^{(1)}, \ldots, m^{(N)}$ is a basis of $M$ (and hence $N=n$ ).

Examples. 1) $\mathbb{C} / G=X^{\left(\mathbb{R}^{n}, M, \mathbb{R}_{+}^{n}\right)}$, where

$$
\tau=\mathbb{R}_{+}^{n}=\left\{x=\left(x_{1}, \ldots, x_{n}\right) \in \mathbb{R}^{n}, x_{i} \geqq 0\right\},
$$

and $M$ can be described as follows. Let $g \in G$, and $r$ be the order of $g$. Then $g$ has the form

$$
\left(x_{1}, \ldots, x_{n}\right) \mapsto\left(\varepsilon_{r}^{a_{1}} x_{1}, \ldots, \varepsilon_{r}^{a_{r}} x_{r}\right),
$$

where $\varepsilon_{r}=\exp \left(\frac{2 \pi i}{r}\right), 0 \leqq a_{i}<r$. Define the lattice

Then

$$
M_{g}=\left\{\left(m_{1}, \ldots, m_{n}\right) \in \mathbb{Z}^{n}: m_{1} a_{1}+\ldots+m_{n} a_{n} \equiv 0 \bmod r\right\} .
$$

$$
M=\bigcap_{g \in G} M_{g} .
$$

It is essential in this construction that $G$ is abelian. To represent the algebra $\mathbb{C}\left[\mathbb{C}^{n} / G\right]$ as the algebra of polynomials whose exponents run over some lattice, 
"the lattice of invariants," one must diagonalize the action of $G$, and it is possible only if $G$ is abelian.

2) $\mathbb{C}^{n}=X^{\left(\mathbb{R}^{n}, \mathbb{Z}^{n}, \mathbb{R}_{+}^{n}\right)}$.

3) $\mathbb{C}^{*} \times \mathbb{C}^{*} \times \ldots \times \mathbb{C}^{*}=X^{\left(\mathbb{R}^{n}, \mathbb{Z}^{n}, \mathbb{R}^{n}\right)}$ (multiplicative torus).

4) A three-dimensional quadratic cone $Q \subset \mathbb{C}^{4}$. Put $Q=X^{\left(\mathbb{R}^{3}, \mathbb{Z}^{3}, \tau\right)}$, where $\tau=\left\langle m^{(1)}, m^{(2)}, m^{(3)}, m^{(4)}\right\rangle$ is the cone spanned by four vectors $m^{(1)}=(1,0,0)$, $m^{(2)}=(0,1,0), m^{(3)}=(0,1,1), m^{(4)}=(1,0,1)$. Then $u_{1}=X_{1}, u_{2}=X_{2}, u_{3}=X_{2} X_{3}$, $u_{4}=X_{1} X_{3}$ is the set of generators of the algebra of functions on $Q$. There is the single relation $u_{2} u_{4}=u_{1} u_{3}$, so $t=1, F\left(u_{1}, u_{2}, u_{3}, u_{4}\right)=u_{2} u_{4}-u_{1} u_{3}$. According to formulas (5), (6), we have

$$
Q=\left\{\left(z_{1}, z_{2}, z_{3}, z_{4}\right) \in \mathbb{C}^{4}: z_{2} z_{4}-z_{1} z_{3}=0\right\} .
$$

Let us take now two affine toric varieties $X=X^{(V, M, \tau)}$ and $Y=X^{\left(V^{\prime}, M^{\prime}, \tau^{\prime}\right)}$. To define a map $f: X \rightarrow Y$ is the same thing as to give a map of function algebras in the opposite direction, $f^{*}: \mathbb{C}[Y] \rightarrow \mathbb{C}[X]$. In toric geometry, one deals with maps $f^{*}$ which respect the structure of semigroup algebras, i.e. which are induced by maps of exponent lattices, $M^{\prime} \rightarrow M$. By a morphism of triplets $\left(V^{\prime}, M^{\prime}, \tau^{\prime}\right) \rightarrow(V, M, \tau)$ we shall mean a linear map $\varphi: V^{\prime} \rightarrow V$, such that $\varphi\left(M^{\prime}\right)<M$, $\varphi\left(\tau^{\prime}\right)<\tau$. Then to each morphism of triples $\varphi$ there corresponds the homomorphism of algebras $\mathbb{C}[Y] \rightarrow \mathbb{C}[X]$, which defines the map $f^{\varphi}: X \rightarrow Y$ between toric varieties.

We see that a toric variety depends on a defining triple $(V, M, \tau)$ in a contravariant way; in particular, if the composition of morphisms of triples $\varphi \circ \psi$ is defined, then $f^{\varphi \circ \psi}=f^{\psi} \circ f^{\varphi}$. To describe how $X^{\tau}$ glue together to give a general toric variety, one needs a covariant dependence. One can obtain a covariant description by passing to dual triples, see the next part. To conclude this part, we explain the term "toric variety."

If $X^{\tau}=X^{(V, M, \tau)}$, then the above discussion and example 3) show that there is the natural imbedding of the $n$-dimensional torus (not to be confused with a compact torus!) $\mathbb{T}^{n}=\mathbb{C}^{*} \times \ldots \times \mathbb{C}^{*}$ into $X^{\tau}$ :

$$
f^{\varphi}: \mathbb{T}^{n}=X^{(V, M, V)} \rightarrow X^{(V, M, \tau)} .
$$

The corresponding morphism of triples is induced by the identity on $V$ and reduces to the substitution of a larger cone for $\tau$, namely, the whole $V$. The torus $\mathbb{T}^{n}$ is a group, and one can verify that the action of $\mathbb{T}^{n}$ on itself by shifts can be extended to an action on $X^{\tau}$. It turns out that this is a characteristic property of toric variety: each $n$-dimensional variety $X$ containing the torus $\mathbb{T}^{n}$ in such a way that its action on itself can be extended to an action on the whole $X$, can be obtained by gluing together affine toric varieties along the common open piece $\mathbb{T}^{n}$ [16]. The procedure of this gluing is described in Part 3.

\section{General Toric Varieties}

To describe conditions which are necessary for the gluing to be possible, it is convenient to pass to the dual objects. Let $W=V^{\vee}$ be the dual space,

$$
\begin{gathered}
N=M^{\vee}=\{w \in W:\langle w, m\rangle \in \mathbb{Z} \forall m \in M\} ; \\
\sigma=\tau^{\vee}=\{w \in W:\langle w, v\rangle \geqq 0 \forall v \in \tau\} ; \\
X_{\sigma}=X_{(W, N, \sigma)}=X^{(V, M, \tau)} .
\end{gathered}
$$



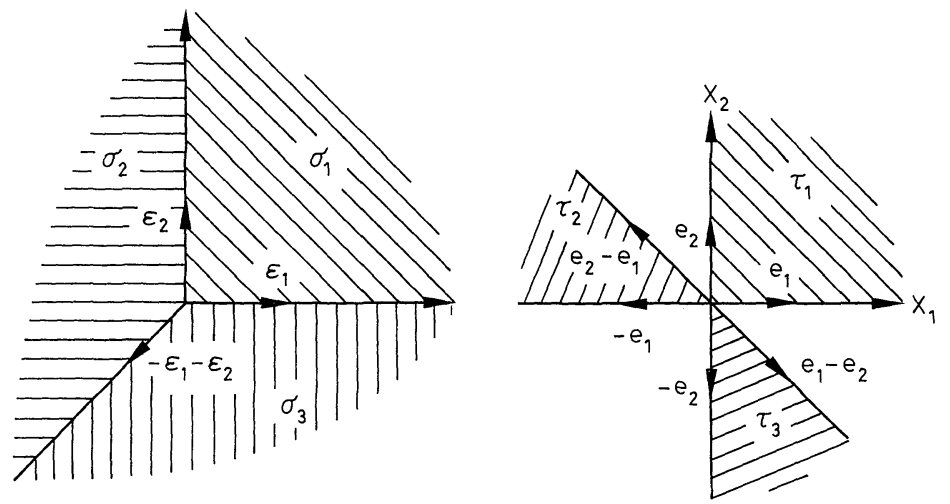

Fig. A1. The dual fan and the basic cones defining coordinate charts for the projective plane

We call a fan in $\mathbb{R}^{n}$ a set $\Sigma$ of cones in $W$, which are rational with respect to $N$ and satisfy the following conditions:

F1. $\sigma_{1}, \sigma_{2} \in \Sigma \Rightarrow \sigma_{1} \cap \sigma_{2}$ is the face of both $\sigma_{1}$ and $\sigma_{2}$.

F2. $\sigma \in \Sigma, \sigma^{\prime} \prec \sigma \Rightarrow \sigma^{\prime} \in \Sigma$ (the record $\sigma^{\prime} \prec \sigma$ means that $\sigma^{\prime}$ is a proper face of $\sigma$ or $\sigma^{\prime}=\sigma$ in which case $\sigma^{\prime}$ is an improper face).

A fan determines the toric variety

$$
X_{(W, N, \Sigma)}=X_{\Sigma}=\bigcup_{\sigma \in \Sigma} X_{\sigma},
$$

in which affine toric pieces $X_{\sigma}$ are glued together along the torus $\left(\mathbb{C}^{*}\right)^{n}=X_{(W, N,\{0\})}$ common for all the $X_{\sigma}$. This rule of gluing pieces $X_{\sigma}$ we shall illustrate by several examples.

Examples. 1) The projective plane $\mathbb{C P}^{2}=X_{\left(\mathbb{R}^{2}, \mathbb{Z}^{2}, \Sigma\right)}$, where

$$
\begin{gathered}
\sum=\left\{\sigma_{j}\right\}_{j=1, \ldots, 7} ; \sigma_{1}=\left\langle e_{1} ; e_{2}\right\rangle, \quad \sigma_{2}=\left\langle e_{2} ;-e_{1}-e_{2}\right\rangle, \\
\sigma_{3}=\left\langle e_{1} ;-e_{1}-e_{2}\right\rangle, \quad \sigma_{4}=\sigma_{3} \cap \sigma_{1}=\left\langle e_{1}\right\rangle, \quad \sigma_{5}=\sigma_{2} \cap \sigma_{1}=\left\langle e_{2}\right\rangle, \\
\sigma_{6}=\sigma_{2} \cap \sigma_{3}=\left\langle-\mathrm{e}_{1}-\mathrm{e}_{2}\right\rangle, \quad \sigma_{7}=\{0\} .
\end{gathered}
$$

The plane is covered by three coordinate charts

(see Fig. A1).

$$
\begin{gathered}
X_{\sigma_{j}}=X^{\tau_{j}} \quad(j=1,2,3), \quad X^{\tau_{j}} \simeq \mathbb{C}^{2}, \\
\mathbb{C}\left[X^{\tau_{1}}\right]=\mathbb{C}\left[\tau_{1} \cap \mathbb{Z}^{2}\right]=\mathbb{C}\left[X_{1}, X_{2}\right], \\
\mathbb{C}\left[X^{\tau_{2}}\right]=\mathbb{C}\left[\tau_{2} \cap \mathbb{Z}^{2}\right]=\mathbb{C}\left[X_{1}^{-1}, X_{2} X_{1}^{-1}\right], \\
\mathbb{C}\left[X^{\tau_{3}}\right]=\mathbb{C}\left[\tau_{3} \cap \mathbb{Z}^{2}\right]=\mathbb{C}\left[X_{2}^{-1}, X_{1} X_{2}^{-1}\right]
\end{gathered}
$$

In each chart we have two affine coordinates $\left(u_{j}, v_{j}\right)$; the transition functions define the projective plane:

$$
\begin{gathered}
\left\{\begin{array} { l } 
{ u _ { 1 } = X _ { 1 } } \\
{ v _ { 1 } = X _ { 2 } }
\end{array} \quad \left\{\begin{array} { l } 
{ u _ { 2 } = X _ { 1 } ^ { - 1 } } \\
{ v _ { 2 } = X _ { 2 } X _ { 1 } ^ { - 1 } }
\end{array} \quad \left\{\begin{array}{l}
u_{3}=X_{2}^{-1} \\
v_{3}=X_{1} X_{2}^{-1}
\end{array}\right.\right.\right. \\
\left\{\begin{array} { l } 
{ u _ { 2 } = u _ { 1 } ^ { - 1 } } \\
{ v _ { 2 } = v _ { 1 } u _ { 1 } ^ { - 1 } }
\end{array} \quad \left\{\begin{array} { l } 
{ u _ { 3 } = u _ { 2 } v _ { 2 } ^ { - 1 } } \\
{ v _ { 3 } = v _ { 2 } ^ { - 1 } }
\end{array} \quad \left\{\begin{array}{l}
u_{1}=v_{3} u_{3}^{-1} \\
v_{1}=u_{3}^{-1} .
\end{array}\right.\right.\right.
\end{gathered}
$$



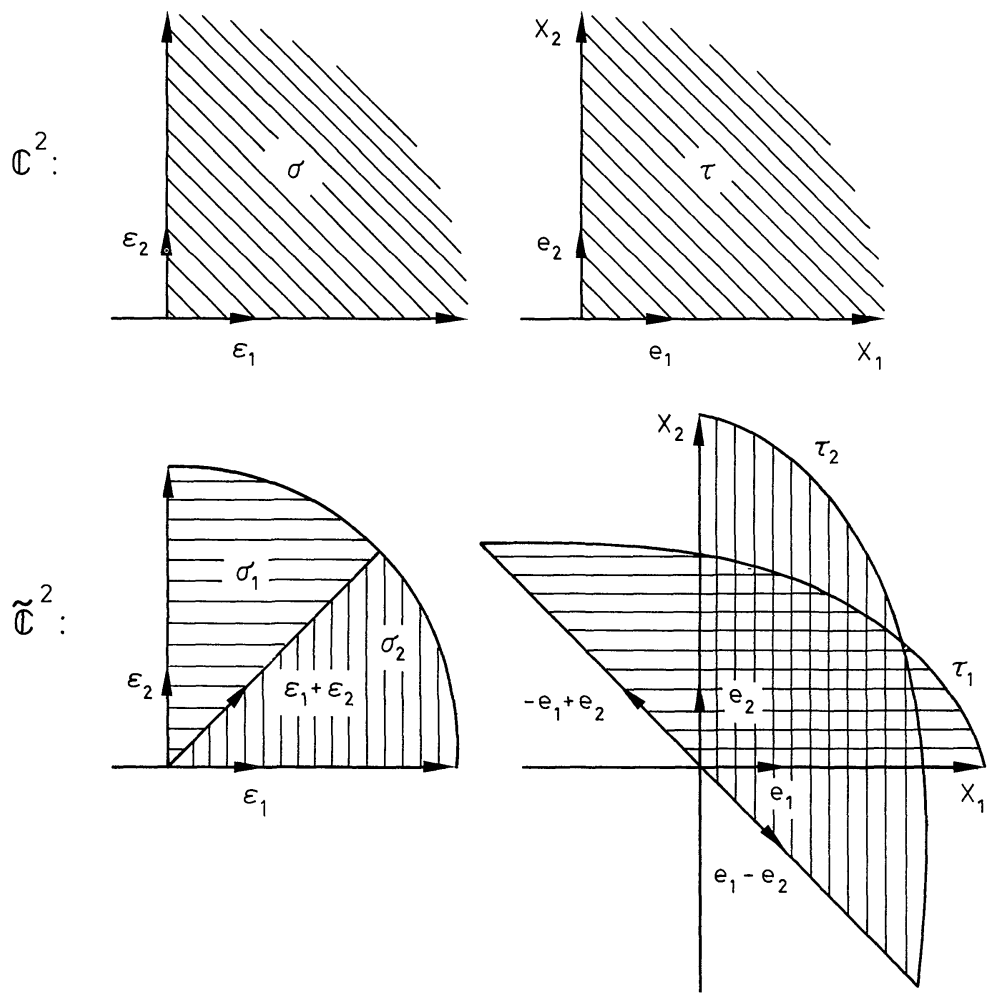

Fig. A2. Blowing up the origin in $\mathbb{C}^{2}$ reduces to subdividing the dual cone $\sigma=\tau^{2}$ into two parts $\sigma_{1}$ and $\sigma_{2}$. The upper row contains toric data for $\mathbb{C}^{2}$, and the lower one for its blow up $\widetilde{\mathbb{C}}_{2}$

2) The fan $\Sigma$ defining the projective space $\mathbb{C P}^{n}$ contains $n+1$ cones,

$$
\begin{gathered}
\sigma_{j}=\left\langle e_{1}, \ldots, e_{j-1},-e_{1}-e_{2}-\ldots-e_{n}, e_{j+1}, \ldots, e_{n}\right\rangle \quad(j=1, \ldots, n), \\
\sigma_{n+1}=\left\langle e_{1}, \ldots, e_{n}\right\rangle,
\end{gathered}
$$

and all the faces of these cones.

3) The fan $\Sigma$ defining the punctured affine space $\mathbb{C}^{n}-\{0\}$ contains $n(n-1)$ dimensional cones

$$
\sigma_{j}=\left\langle e_{1}, \ldots, \hat{e}_{j}, \ldots, e_{n}\right\rangle,
$$

and all their faces, but contains no $n$-dimensional cones.

4) The blow up of $\mathbb{C}^{2}$ at the origin (see Fig. A2).

$$
\begin{aligned}
& \mathbb{C}\left[\tau_{1} \cap \mathbb{Z}^{2}\right]=\mathbb{C}\left[X^{e_{1}}, X^{-e_{1}+e_{2}}\right]=\mathbb{C}\left[X_{1}, X_{2} X_{1}^{-1}\right], \\
& \mathbb{C}\left[\tau_{2} \cap \mathbb{Z}^{2}\right]=\mathbb{C}\left[X^{-e_{2}+e_{1}}, X^{e_{2}}\right]=\mathbb{C}\left[X_{2}, X_{1} X_{2}^{-1}\right] .
\end{aligned}
$$

We have:

$$
\begin{aligned}
& \left\{\begin{array} { l } 
{ u _ { 1 } = X _ { 1 } } \\
{ v _ { 1 } = X _ { 2 } X _ { 1 } ^ { - 1 } }
\end{array} \quad \left\{\begin{array}{l}
u_{2}=X_{2} \\
v_{2}=X_{1} X_{2}^{-1}
\end{array}\right.\right. \\
& \left\{\begin{array} { l } 
{ u _ { 2 } = u _ { 1 } v _ { 1 } } \\
{ v _ { 2 } = v _ { 1 } ^ { - 1 } }
\end{array} \quad \left\{\begin{array}{l}
u_{1}=u_{2} v_{2} \\
v_{1}=v_{2}^{-1} .
\end{array}\right.\right.
\end{aligned}
$$


Formulas (13) are those of the $\sigma$-process (or blow up) $\widetilde{\mathbb{C}}^{2} \rightarrow \mathbb{C}^{2}$.

Proposition 2. Let $(W, N, \Sigma) \stackrel{a}{\rightarrow}\left(W^{\prime}, N^{\prime}, \Sigma^{\prime}\right)$ be a morphism of triples, i.e. a linear map: $W \rightarrow W^{\prime}$ such that $\alpha(N) \subset N^{\prime}$, and for every cone $\sigma \in \Sigma$ there exists a cone $\sigma^{\prime} \in \Sigma^{\prime}$ with $\alpha(\sigma) \subset \sigma^{\prime}$. Then $\alpha$ induces the holomorphic map of toric varieties $f_{\alpha}: X_{(W, N, \Sigma)} \rightarrow X_{\left(W, N^{\prime}, \Sigma^{\prime}\right)}$. Furthermore:

(i) The map $f_{\alpha}$ is surjective and has compact fibers if and only if $\left|\Sigma^{\prime}\right|=\alpha^{-1}(|\Sigma|)$,

$|\Sigma|$ denoting the support of the fan, $|\Sigma|=\bigcup_{\sigma \in \Sigma} \sigma$.

(ii) The map $f_{\alpha}$ is a resolution of singularities, if $\alpha$ is an isomorphism of lattices, $\alpha:|\Sigma| \rightarrow\left|\Sigma^{\prime}\right|$ is bijective and all cones in $\Sigma$ are basic.

For proof see [15].

Corollary. The variety $X_{\Sigma}$ is compact if and only if $|\Sigma|=W$ (such a fan is said to be complete).

Proof. Apply part (i) of Proposition 2 to the case when $W=W^{\prime}, N=N^{\prime}, \Sigma^{\prime}=\left\{\mathbb{R}^{n}\right\}$ (the map to a point).

\section{Resolution of Singularities}

From Proposition 2, one sees that resolving singularities of $X_{\sigma}$ is equivalent to dividing $\sigma$ into basic cones (resolving singularities is a local procedure, so it suffices to know the structure of resolutions of affine toric varieties $X_{\sigma}$ ). This problem is purely combinatorial.

Examples. 1) Resolution of the three-dimensional quadratic singularity $0 \in Q \subset \mathbb{C}^{4}$, see Example 4) from part 2. There are three variants; one can divide $\sigma$ into four basic cones by the ray spanned by the vector $(1 ; 1 ; 1)$, or into two basic cones by any of the two planes passing through two opposite edges of $\sigma$. The first way increases the number of one-dimensional cones in $\Sigma$ by 1 ; below we shall see that this leads to the increase of the number of divisors in $X_{\Sigma}$, i.e. that some divisor (subvariety of codimension 1) has been glued in place of the singular point 0 . In the second way, we glue in some subvariety of codimension 2 , or a curve in the case under consideration, in place of 0 (this corresponds to increasing the number of two-dimensional cones in the fan).

2) Quotient $\mathbb{C}^{2} / \Gamma$ under a cyclic group $\Gamma$ of order $r$, whose generator acts by the formula

$$
c:\left(z_{1}, z_{2}\right) \mapsto\left(\varepsilon_{r}^{a_{1}} z_{1}, \varepsilon_{r}^{a_{2}} z_{2}\right) .
$$

Consider the action $\frac{1}{r}(1, r-1)$ (i.e. $\left.a_{1}=1, a_{2}=r-1\right)$. The invariant lattice (9) has the basis $\varepsilon_{1}=(r, 0), \varepsilon_{2}=(-r+1,1)$. In this basis, the cone $\tau$ is the cone spanned by two vectors $\varepsilon_{1}$ and $r \varepsilon_{2}+(r-1) \varepsilon_{1}$. The dual cone $\sigma$ is spanned by two vectors $r \check{\varepsilon}_{1}-(r-1) \check{\varepsilon}_{2}, \check{\varepsilon}_{2}$, and it can be divided into $r$ basic cones by vectors $\check{\varepsilon}_{1}$, $2 \check{\varepsilon}_{1}-\check{\varepsilon}_{2}, \ldots,(r-1) \check{\varepsilon}_{1}-(r-2) \check{\varepsilon}_{2}$ (see Fig. A3). This resolution glues in $r-1$ curves $E_{j} \simeq \mathbb{C \mathbb { P } ^ { 1 }}$, and the intersection matrix $\left(E_{i} \cdot E_{j}\right)$ is equal to the Cartan matrix of the algebra $\mathscr{A}_{r-1}$ taken with the opposite sign (this is the reason for which the singularity $\mathbb{C}^{2} / \Gamma$ is said to be of type $\left.\mathscr{A}_{r-1}\right)$.

3) When constructing the Calabi-Yau space (CYS) from the 8-th string one must resolve the singularity $\mathbb{C}^{3} / \mathbb{Z}_{7}$ of the form $\frac{1}{7}(1,2,4)$. Similar to the preceding 

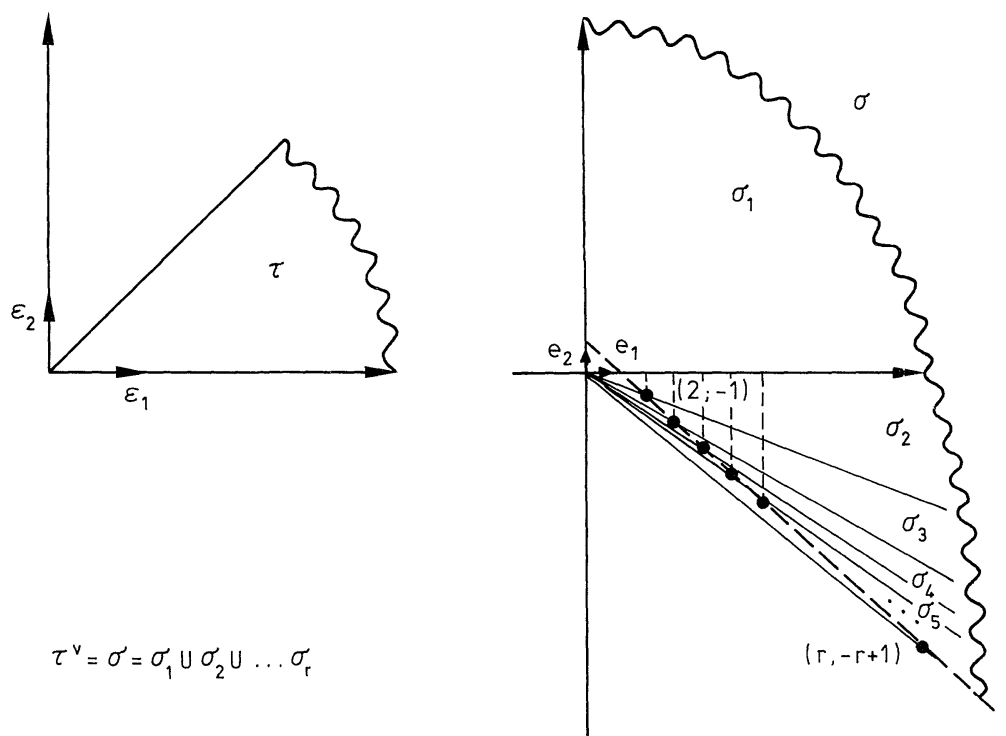

Fig. A3. Blowing up the $A_{r-1}$-singularity. The defining cone $\tau$ in the exponent plane $\mathbb{R}^{2}$ is on the left. On the right we plot at the same figure the dual cone $\sigma$ and its subdivision by $r$ basic cones defining a resolution of the $A_{r-1}$-singularity

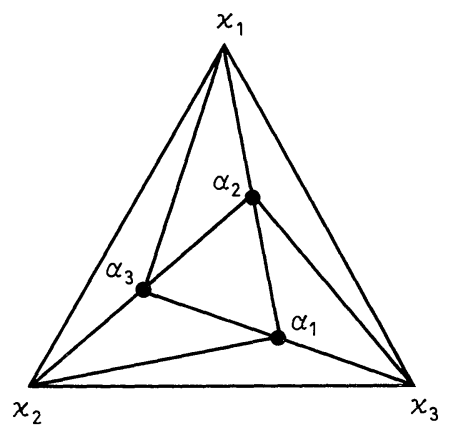

Fig. A4. The triangle $\kappa_{1} \kappa_{2} \kappa_{3}$ is the section of the dual cone $\sigma$ defining the singularity of type $\frac{1}{7}(124)$. The smaller triangles are the trace of the subdivision of $\sigma$ by basic cones. This subdivision defines a resolution of the singularity

example, we choose a basis of $M$ in the form

$$
\left(\varepsilon_{1}, \varepsilon_{2}, \varepsilon_{3}\right)=\left(\begin{array}{rrr}
7 & -2 & -4 \\
0 & 1 & 0 \\
0 & 0 & 1
\end{array}\right) \text {. }
$$

Then we have in the basis $\left(\check{\varepsilon}_{1}, \check{\varepsilon}_{2}, \check{\varepsilon}_{3}\right)$ :

$$
\sigma=\left\langle\kappa_{1}, \kappa_{2}, \kappa_{3}\right\rangle
$$

where

$$
\left.\left(\kappa_{1}, \kappa_{2}, \kappa_{3}\right)=\begin{array}{rrr}
7 & 0 & 0 \\
-2 & 1 & 0 \\
-4 & 0 & 1
\end{array}\right) .
$$




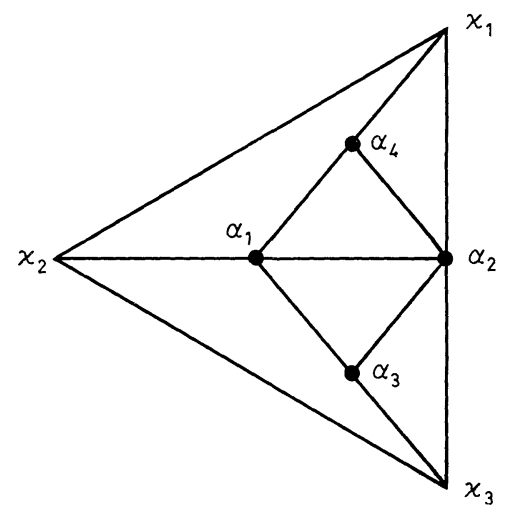

Fig. A5. The trace of the fan defining a resolution of the singularity of type $\frac{1}{8}(125)$

Let us draw the trace of the fan, resolving the singularity, in the plane $H=\left(\kappa_{1}, \kappa_{2}, \kappa_{3}\right)($ Fig. A4):

$$
\begin{gathered}
\alpha_{1}=(1 ; 0 ; 0)=\frac{1}{7}\left(\kappa_{1}+2 \kappa_{2}+4 \kappa_{3}\right) \in H \cap N, \\
\alpha_{2}=(4 ;-1 ;-2)=\frac{1}{2}\left(\alpha_{1}+\kappa_{1}\right) \in H \cap N, \\
\alpha_{3}=(2 ; 0 ;-1)=\frac{1}{7}\left(2 \kappa_{1}+4 \kappa_{2}+\kappa_{3}\right) \in H \cap N .
\end{gathered}
$$

This resolution glues in three divisors $E_{\alpha_{1}}, E_{\alpha_{2}}, E_{\alpha_{3}}$, and $X_{\Sigma}$ is covered by the seven coordinate charts

$$
X_{\sigma_{j}} \simeq \mathbb{C}^{3} \quad(j=1, \ldots, 7) \text {. }
$$

4) The singularity $\frac{1}{8}(1 ; 2 ; 5)$ is resolved similarly; we give the result. Here we have four divisors glued in and eight coordinate charts (Fig. A5)

$$
\left(\kappa_{1}, \kappa_{2}, \kappa_{3}\right)=\left(\begin{array}{rrr}
8 & 0 & 0 \\
-2 & 1 & 0 \\
-5 & 0 & 1
\end{array}\right) ; \quad \begin{array}{ll}
\alpha_{1}=\frac{1}{2}\left(\kappa_{2}+\alpha_{2}\right), & \alpha_{2}=\frac{1}{2}\left(\kappa_{1}+\kappa_{3}\right), \\
\alpha_{3}=\frac{1}{2}\left(\alpha_{1}+\kappa_{3}\right), & \alpha_{4}=\frac{1}{2}\left(\alpha_{1}+\kappa_{1}\right) .
\end{array}
$$

Proposition 3. Let $X$ be a toric variety. Let $\kappa_{1}, \ldots, \kappa_{N}$ be the shortest integer vectors on one-dimensional edges of $\sigma$. Suppose that $\sigma$ is divided into basic cones, and denote the shortest integer vectors lying on one-dimensional edges of this partition, other than edges of $\sigma$, through $\alpha_{1}, \ldots, \alpha_{k}$. Then there is a natural way to associate the divisors $D_{\alpha_{j}}$ on $X_{\Sigma}$ to the vectors $\alpha_{j}$.

(i) There is a holomorphic nowhere-vanishing exterior form of the highest degree on $X_{\sigma}$ if and only if the endpoints of $\kappa_{1}, \ldots, \kappa_{N}$ lie in a hyperplane $H$ and the lattice $H \cap N+\mathbb{Z} \kappa_{1}$ coincides with $N$.

(ii) Suppose that $\omega \in \Gamma\left(X_{\sigma}, \Omega_{X_{\sigma}}\right)$ is a nowhere-vanishing holomorphic form, $H$ the hyperplane defined in $(i),\left\langle w, m_{H}\right\rangle=1$ its equation, and $\tilde{\omega}$ the pullback of $\omega$ on $X_{\Sigma}$. Then

$$
\operatorname{ord}_{D_{\alpha_{j}}}(\tilde{\omega})=\left\langle\alpha_{j}, m_{H}\right\rangle-1
$$

In particular, $\tilde{\omega}$ has no zeros on $X_{\Sigma}$ if and only if $\alpha_{j} \in H$ for all $j=1, \ldots, k$. 
Proof. The torus $\mathbb{T}^{n}=\left(\mathbb{C}^{*}\right)^{n}$ acts on any toric variety $X_{\Sigma}$, since it acts on each open piece $X_{\sigma}$ and these actions coincide on intersections of $X_{\sigma}$. All the orbits of $\mathbb{T}^{n}$ are also tori. To be more precise, to each cone $\sigma \in \Sigma$, one associates the unique closed orbit of $\mathbb{T}^{n}$ in $X_{\sigma}$, namely, the closed subvariety $X^{L(\check{\sigma})}$, where $L(\check{\sigma})$ denotes the ridge of $\check{\sigma}$, that is the largest linear space contained in $\check{\sigma}$. It is obvious, that $\operatorname{dim} \sigma+\operatorname{dim} L(\check{\sigma})=n$, so the $k$-dimensional cones in $\sigma$ are in $1-1$ correspondence with the $(n-k)$-dimensional orbits in $X_{\Sigma}$. We use the notation $\mathcal{O}_{\sigma}=X^{L(\check{\sigma})}$, and by $F_{\sigma}$ we denote the closure of $\mathscr{O}_{\sigma}$ in $X_{\Sigma}$. Then divisors $D_{\alpha_{J}}$ are defined by $D_{\alpha_{J}}=F_{\sigma_{J}}$, where $\sigma_{j}=\mathbb{R}_{+} \alpha_{j}$ are one-dimensional cones in $\Sigma$.

To prove (i), we note, that by Proposition 1, (iii) every differential on $X_{\sigma}$ has the form $\sum a_{m} X^{m} \omega_{0}$, where the sum is taken over $m \in \tau^{0}$ and almost all $a_{m}=0$. The existence of a differential $\omega \in \Gamma\left(\Omega_{X_{\sigma}}\right)$ without zeros on $X_{\sigma}$ is equivalent to the following property: the semigroup $\tau^{0} \cap M$ has the "smallest" element $m^{\prime}$, such that

Put

$$
\tau^{0} \cap M=m^{\prime}+\tau \cap M .
$$

$$
H=\left\{w \in W:\left\langle w, m^{\prime}\right\rangle=1\right\},
$$

and verify that $\kappa_{j} \in H$. Let $\kappa=\kappa_{j}, \tau_{0}$ the face of $\tau$ orthogonal to $\kappa$. We want to study the behavior of $\omega$ in the neighbourhood of the divisor $D_{\kappa}$. It is clear that we can conisder instead of $X^{\tau}$ any open subset having non-zero intersection with $D_{\kappa}$. In particular, we can pass to the open subvariety $X^{\bar{\tau}} C X^{\tau}$, where $\bar{\tau}$ is the half-space $\mathbb{R} \tau_{0}+\mathbb{R}_{+} \tau$. Then $D_{\kappa}=X^{\bar{\tau}_{0}}$ where $\bar{\tau}_{0}=L(\bar{\tau})$ is the ridge of $\bar{\tau}$, and the dual cone $\bar{\sigma}=\mathbb{R}_{+} \kappa$ is one-dimensional. Choosing another basis for our lattice, we come to the following, putting:

$$
\begin{gathered}
\bar{\tau}=\left\{\kappa \in \mathbb{R}^{n}: x_{n} \geqq 0\right\}, \quad \bar{\tau}_{0}=\left\{x_{n}=0\right\}, \\
X^{\bar{\tau}}=X^{\left(\mathbb{R}^{n}, \mathbb{Z}^{n}, \bar{\tau}\right)} \simeq\left(\mathbb{C}^{*}\right)^{n-1} \times \mathbb{C}=\left\{\left(z_{1}, \ldots, z_{n}\right) \in \mathbb{C}^{n}: z_{1} \cdot z_{2} \cdot \ldots \cdot z_{n-1} \neq 0\right\} .
\end{gathered}
$$

Then $\mathscr{D}_{\kappa}=\left(\mathbb{C}^{*}\right)^{n-1}=\left\{z_{n}=0\right\}$. The order of vanishing of the differential $z^{m^{\prime}} \frac{d z_{1}}{z_{1}} \wedge \ldots \wedge \frac{d z_{n}}{z_{n}}$ on $D_{\kappa}$ is equal to $m_{n}^{\prime}-1$. By choosing $\kappa=(0 ; 0 ; \ldots ; 1)$, the nonvanishing of $\omega=z^{m^{\prime}} \cdot \omega_{0}$ on $D_{\kappa}$ is equivalent to the equality $\left\langle\kappa, m^{\prime}\right\rangle=m_{n}^{\prime}=1$, and this means that $\kappa \in H$. As the assertion is true for any $\kappa_{j}$, the first part of the proposition is proved. The assertion about zeros of differentials on $D_{\alpha_{j}}$ is proved similarly.

Corollary. In Examples 2)-4) the canonical bundle of the variety $X_{\Sigma}$ resolving singularities of $X_{\sigma}$ is trivial, that is, there is a nowhere-vanishing differential on $X_{\Sigma}$.

\section{Resolution of Singularities which does not Affect the Canonical Class}

Suppose we are given a linear action of an abelian group $G$ on the affine space $\mathbb{C}^{n}$. We can take without loss of generality this action to be diagonal. The quotient map $\mathbb{C}^{n} \rightarrow \mathbb{C}^{n} / G$ corresponds to the choice of the invariant lattice $M \subset M_{0}$, defined by formula (9), in the lattice $M_{0}=\mathbb{Z}^{n}$, defining $\mathbb{C}^{n}=X^{\left(\mathbb{R}^{n}, M_{0}, \mathbb{R}^{n}\right)}$ [the morphism of triples $\left(\mathbb{R}^{n}, M, \mathbb{R}_{+}^{n}\right) \mapsto\left(\mathbb{R}^{n}, M_{0}, \mathbb{R}_{+}^{n}\right)$ is the identity on $\mathbb{R}^{n}$ and defines the inclusion of lattices $M \hookrightarrow M_{0}$, see Part 2]. It is obvious that $\left[M_{0}: M\right]=|G|$. For dual lattices, $\left[N: N_{0}\right]=|G|$. 
Proposition 4. Suppose the following properties are satisfied: a) G contains no quasireflections, i.e. elements whose fixed set is of codimension 1 ; b) $G$ is unitary, i.e. in (8) we have $\left.\sum a_{i} \equiv 0(\bmod r) ; \mathrm{c}\right) n=2$ or 3 . Then the quotient $\mathbb{C}^{n} / G$ has a resolution with trivial canonical class.

Proof. Apply Proposition 2. We have $N=H \cap N+\mathbb{Z} \kappa_{1}$. Hence $\left[H \cap N: H \cap N_{0}\right]$ $=|G|$, since $\kappa_{1} \in N_{0}$. As the triangle (for $n=3$ ) of the segment (for $n=2$ ), $H \cap \sigma$, contains no points of the lattice $N_{0}$, this triangle (segment) can be divided into $|G|$ smaller triangles (segments) which do not contain points of $N$ other than their vertices. Consequently, their sides will give us bases of $N \cap H$ (here it is essential that $n \leqq 3$ ). So, the cones spanned by these triangles will be basic, and they will form the fan $\sum$ resolving the singularities of $\mathbb{C}^{n} / G$. As all the ends of vectors dividing $\sigma$ are in $H$, the canonical bundle of $X_{\Sigma}$ is trivial (by Proposition 3).

We state separately the following fact:

Corollary. The fan $\Sigma$ resolving singularities in Proposition 4 contains exactly $|G|$ cones of the maximal dimension (equal to $n$ ).

\section{Fundamental Group}

Proposition 5. Let $\left(\mathbb{R}^{n}, N, \Sigma\right)$ be a triple defining a toric variety $X_{\Sigma}$. For each $\sigma \in \Sigma$, denote by $\mathbb{R} \sigma$ the linear subspace in $\mathbb{R}^{n}$ spanned by $\sigma$. Then $\pi_{1}\left(X_{\Sigma}\right)=N / N_{\Sigma}$, where

$$
N_{\Sigma}=\sum_{\sigma \in \Sigma} \mathbb{R} \sigma \cap N
$$

(the sum on the right stands for the sum of subgroups of the group $N$ ). In particular, $X_{\Sigma}$ is simply connected if $\Sigma$ contains at least one cone of dimension $n$.

Proof. See [15].

\section{References}

1. Green, M.B., Schwarz, J.H.: Supersymmetrical dual string theory.Nucl. Phys. B 181, 502 (1981); Supersymmetric dual string theory, II. Vertices and trees, and III. Loops and renormalization. B 198, 252 (1982); B 198, 441 (1982); Supersymmetrical string theories. Phys. Lett. 109 B, 444 (1982); Anomaly cancellations in supersymmetric $D=10$ gauge theory and superstring theory. $149 \mathrm{~B}, 117$ (1984)

Green, M.B., Schwarz, J.H., Brink, L.: $N=4$ Yang-Mills and $N=8$ supergravity as limits of string theories. Nucl. Phys. B 198, 474 (1982)

2. Gross, D.J., Harvey, J.A., Martinec, E., Rohm, R.: Heterotic string. Phys. Rev. Lett. 54, 502 (1985)

3. Candelas, P., Horowitz, G., Strominger, A., Witten, E.: Vacuum configurations for superstrings. Nucl. Phys. B 256, 46 (1985)

4. Witten, E.: New issues in manifolds of SU(3) holonomy. Preprint, Princeton, October 1985

5. Witten, E.: Symmetry breaking patterns in superstring models. Nucl. Phys. B 258, 75 (1985)

6. Strominger, A., Witten, E.: New manifolds for superstring compactification. Commun. Math. Phys. 101, 341 (1985)

Strominger, A.: Topology of superstring compactification HSF-ITP-85-109; Yukawa couplings in superstring compactification HSF-ITP-85-105 
7. Yau, S.-T.: Compact three dimensional Kähler manifolds with zero Ricci curvature. In: Proceedings of the Argonne symposium on anomalies, geometry and topology. Bardsen (ed.), 1985, p. 395

8. Griffiths, P., Harris, J.: Principles of algebraic geometry. New York: Wiley 1978

9. Dixon, L., Harvey, J.A., Vafa, E., Witten, E.: Strings on orbifolds. Nucl. Phys. B 261, 678 (1985); Strings on orbifolds, (II). B 274, 285 (1986)

10. Kac, V.: Adv. Math. Infinite-dimensional algebras, Dedekind's $\eta$-function, classical Möbious function and very strange formula 30, 85 (1978)

11. Bourbaki, N.: Groupes et algèbres de Lie, Chaps. IV-VI. Paris. Hermann 1968

12. Yau, S.-T.: On the Ricci curvature of compact Kähler manifolds and complex MongeAmpère equations I. Commun. Pure Appl. Math. 31, 339 (1978)

13. Beauville, A.: Variétés kähleriennes dont la première classe de Chern est nulle. J. Differ. Geom. 18, 755 (1983); Some remarks on Kähler manifolds with $c_{1}=0$. Proc. Katata Conf., Katata 1983, p. 1

14. Popov, V.L.: Discrete complex reflexion groups. Commun. Math. Inst. Utrecht No. 15, 1982; Shepard, G.C., Todd, J.A.: Canad. J. Math. 6, 274 (1954)

15. Danilov, V.I.: Geometry of toric varieties. Usp. Mat. Nauk 33, 73 (1978) (in Russian) Kempf, J., Knudsen, F., Mumford, D., Saint-Donat, B.: Toroidal embeddings. I. Lecture Notes in Mathematics, Vol. 339. Berlin, Heidelberg, New York: Springer 1973

16. Hovansky, A.G.: Newton polyhedra (resolution of singularities). Sovremen. Probl. Mat. 22 M. (1983) (in Russian)

Communicated by Ya. G. Sinai

Received September 10, 1986 